\title{
Programming at the Edge of Synchrony
}

\author{
CEZARA DRĂGOI, INRIA, ENS, CNRS, PSL ${ }^{*}$, France and Informal Systems, France \\ JOSEF WIDDER, Informal Systems, Austria \\ DAMIEN ZUFFEREY, Max Planck Institute for Software Systems, Germany
}

\begin{abstract}
Synchronization primitives for fault-tolerant distributed systems that ensure an effective and efficient cooperation among processes are an important challenge in the programming languages community. We present a new programming abstraction, RESYNC, for implementing benign and Byzantine fault-tolerant protocols. RESYNC has a new round structure that offers a simple abstraction for group communication, like it is customary in synchronous systems, but also allows messages to be received one by one, like in the asynchronous systems. This extension allows implementing network and algorithm-specific policies for the message reception, which is not possible in classic round models.

The execution of RESynC programs is based on a new generic round switch protocol that generalizes the famous theoretical result about consensus in the presence of partial synchrony by of Dwork, Lynch, and Stockmeyer. We evaluate experimentally the performance of RESYNC's execution platform, by comparing consensus implementations in RESynC with LiBPAXos3, ETCD, and BFT-SMART, three consensus libraries tolerant to benign, resp. byzantine faults.
\end{abstract}

CCS Concepts: • Software and its engineering $\rightarrow$ Domain specific languages; Control structures; Runtime environments; • Theory of computation $\rightarrow$ Distributed computing models.

Additional Key Words and Phrases: Distributed systems, Fault-tolerance, Round Model, Synchrony

ACM Reference Format:

Cezara Drăgoi, Josef Widder, and Damien Zufferey. 2020. Programming at the Edge of Synchrony. Proc. ACM Program. Lang. 4, OOPSLA, Article 213 (November 2020), 30 pages. https://doi.org/10.1145/3428281

\section{INTRODUCTION}

Fault-tolerant distributed algorithms are notorious for being hard to design and implement. One needs to take into account features of a non-deterministic network, behaviors of faulty processes, as well as the asynchrony of the actions performed by correct processes. These algorithms underpin many distributed applications and critical services, e.g., Zab [Junqueira et al. 2011], Zyzzyva [Kotla et al. 2009] or more recent blockchains like Tendermint [Buchman 2016] or Libra [Baudet et al. 2019]. These systems are implemented in general-purpose programming languages that support the asynchronous programming paradigm. This programming paradigm is error-prone and facilitates bug occurrences [CASSANDRA 2013; Sutra 2019; ZOOKEEPER 2017] even when implementing protocols that have been formalized and proved like Paxos [Lamport 2005]. These bugs show that going from (verified) protocols to implementations is a difficult task. The asynchronous programming paradigm, despite its pitfalls, is preferred because it enables important performance optimizations but also because other suitable programming abstractions and synchronization

Authors' addresses: Cezara Drăgoi, INRIA, ENS, CNRS, PSL*, France, cezara.dragoi@ens.fr, Informal Systems, France, cezara@informal.systems; Josef Widder, Informal Systems, Austria, josef@informal.systems; Damien Zufferey, Max Planck Institute for Software Systems, Kaiserslautern, Germany, zufferey@mpi-sws.org.

This work is licensed under a Creative Commons Attribution 4.0 International License.

(c) 2020 Copyright held by the owner/author(s).

2475-1421/2020/11-ART213

https://doi.org/10.1145/3428281

Proc. ACM Program. Lang., Vol. 4, No. OOPSLA, Article 213. Publication date: November 2020. 
primitives are lacking. Therefore, our goal is to identify new synchronization primitives that simplify programming distributed systems, tolerant to benign and byzantine faults.

The seminal work [Dwork et al. 1988] defines the basic round model, the first abstract computational model that offers rounds as a synchronous primitive. The model establishes its notoriety because it can solve consensus, i.e., $n$ processes trying to agree on a value, provided that the network satisfies the partial synchrony assumption. Consensus is not solvable over asynchronous networks in the presence of faults [Fischer et al. 1985] and partial synchrony is one of the weakest theoretical assumptions that allows us to solve it. Partial synchrony means that the network eventually becomes synchronous, i.e., there exists a bound $\Delta$ on the message delay and a bound $\Phi$ on the relative speed of processes, however these bounds hold only from some time on, called global stabilization time GST. (There are two other definitions of partial synchrony in [Dwork et al. 1988], which are less used.) The basic round model, and the follow-up round-based approaches [Charron-Bost and Schiper 2009; Drăgoi et al. 2016; Gafni 1998], rely on an abstract global clock. The abstract global clock synchronizes all processes, that is, all processes execute the code of a round in lockstep (at the same time), where a round is a sequence of sending messages, receiving (some or all) messages sent in that round in one atomic step, followed by some local updates based on the received messages. To "execute" a consensus-solving algorithm in a round model, one has to implement an algorithm that computes when the round switch should locally happen such that processes switch rounds at the same time. To this, Dwork et al. [1988] use an algorithm that implements a distributed clock, exploiting $\Delta$ and $\Phi$ from the partially synchronous network assumption. The distributed clock determines the round switch at each process. Roughly, before GST the system is in an arbitrary state (different processes may have different clock values), but after GST, the distributed clock is guaranteed to simulate the abstract global clock and implicitly synchronizes all the correct processes. This is a purely theoretic result, since it requires multiple synchronization steps (clock steps) within a round, and it makes simplistic assumptions regarding the time spent by processes sending and receiving messages or performing local computation. In general, round-based solutions are infamous for their prohibitive performance, although most existing solutions for consensus rely on the partial synchrony assumptions and implicitly on rounds.

Among the systems that rely on partial synchrony are PBFT [Castro and Liskov 2002], BftSmart[Bessani et al. 2014], ViewStamped Replication [Oki and Liskov 1988], Zookeeper's atomic broadcast protocol [Junqueira et al. 2011], Apache Cassandra [Lakshman and Malik 2010], libPaxos [Sciascia 2016], or the more recent works emerging from blockchain research like Tendermint [Buchman et al. 2018], RedBelly [Crain et al. 2018], Lumière [Naor et al. 2019], Hot-Stuff [Yin et al. 2019], or Libra [Baudet et al. 2019]. Each of these systems, use rounds implicitly and propose a new algorithm for the round switch that exploits partial synchrony in a different way, thus resulting in a new solution for consensus (state machine replication) either in the benign, or in the Byzantine case. Although solving the same problem under similar network assumptions, these are stand-alone systems, sharing no high-level control structure. They are implemented in error prone asynchronous programming paradigms (as shown by numerous bug reports), which are preferred for facilitating performance optimizations. These systems exploit the synchronicity of the network after GST differently, and they guarantee that soon after GST, correct processes are synchronized (a property called eventual synchrony [Dutta et al. 2005]), and reach consensus. To implement eventual synchrony, these systems use asynchronous features like properties on the set of received messages (e.g., a message with a certain payload or from a certain sender has been received, or a certain number of messages have been received) that trigger a local round switch or timeouts on the local duration of a round, which ensure that after GST the processes get synchronized. These features are opaque in the basic round model as they are hidden in the theoretical implementation of the distributed clock. In the basic-round model, and in all follow-up round-based programming 
abstractions, processes have no control over the set of received messages and no control over the round switch.

Problem statement. In this paper we ask the following question: is there a programming abstraction that systematizes the optimization principles used in implementations of consensus protocols for partially synchronous networks? More widely, is there a synchronous round-based model that allows protocol specific optimizations, which today are possible only in asynchronous programming frameworks?

We propose RESYNC, a new programming language with a novel round structure which preserves the abstraction level of theoretical round-based models and reasonably matches the performance demands of large-scale systems like BFt-SMARt [Bessani et al. 2014] or LiBPAxos3 [Sciascia 2016] RESYNC is a parametric framework that accommodates various implementations of the eventual synchrony property, rather than a fixed implementation, as it is the case in all previous works. We systematize the optimization principles used in large-scale systems that solve consensus and wrap them into a domain-specific language, that facilitates future consensus implementations under partial synchrony, and in general facilitates exploiting the network specifics.

RESYNC is addressed to the algorithm designer who wants to prototype algorithms without worrying about network code and data management, and to the programmer less versatile in reasoning about interleavings and partial synchrony.

Key insights. In synchronous (or round-based) models, computations are structured in a sequence of rounds, where in each round all processes send messages and then update their local state based on the set of received messages. Rounds are communication-closed [Elrad and Francez 1982] and are a powerful synchronization primitive: messages are not received outside the round they were sent. The message reception of a round is hidden from the protocol's perspective: a non-deterministically chosen subset of the messages sent is received in one atomic step. Round structures are known for their poor performance due to an excessive need of synchronization. Any execution framework for the round-structure must implement a round switch algorithm. All known approaches are either too general [Dwork et al. 1988] and hence purely theoretical due their poor performance, or they are restricted to a sub-class of distributed protocols like PSYNC [Drăgoi et al. 2016] which implements one solution for eventual synchrony (that is not customizable). It is well established that there is no unique round switch algorithm that fits the performance needs of all protocols. Therefore, to design a more flexible way to implement the round switch we turn to asynchronous computational models.

Asynchronous computational models are preferred for implementing systems due to their better performance. Still, algorithm designers, present their asynchronous solutions in terms of rounds, phases, epochs, ballots, etc., e.g., [Dwork et al. 1988; Lamport 2005; Ongaro and Ousterhout 2014]. These notions encode some logical time that allows to structure and decompose distributed computations along the time line. Although presented in rounds, the system is implemented under the asynchronous semantics because it enables network-specific performance optimizations: messages are received one-by-one, which allows the system to react quickly to incoming messages, as opposed to the round structure that would wait for all the messages of the current round before reacting. For example, under the asynchronous semantics a process could switch rounds fast, as soon as it receives a message that contains the information it was waiting for, e.g., the first message that is receives comes from the leader and contains the value all processes agree on. In contrast, when executing the synchronous round-based model, a process would wait for all the messages sent in that round, even if the followers (the processes that are not leaders) only relay the message from the leader for fault-tolerance purposes. 
The main insight is that we want a round structure where messages are received one by one, instead of all in one atomic step. Therefore, we define a new round structure where a round combines:

\section{Sending messages,}

A message accumulator that is a new round component which allows programming the message reception within the round-based model, and computes the sequence of messages received in a round, and

A round computation that updates the local state, depending on the mailbox of the current round computed by the message accumulator.

The executions of the new round model preserve the lockstep semantics at the round boundaries, but, within a round, messages are received asynchronously by the message accumulators of different processes. In this way, messages are received one-by-one and the accumulator decides when to wait for more messages or transition to the next round. This removes the need for extra synchronization, i.e., synchronization barriers needed to satisfy the (conservative) guarantees of the round model but which are not required for the protocol-specific progress condition.

The implementation of the message accumulator, more precisely the termination of a message accumulator, uses new features that are not available in any other round-based programming abstraction. First, the language provides constructs to control the termination of a message accumulator using timeouts, or using properties satisfied by the received messages for the current round. These features are sufficient to program message accumulators for distributed protocols whose specification is solvable in asynchronous networks.

However, timeouts and conditions on the set of messages received for the current round are not sufficient for implementing a round-switch in consensus protocols. The implementations of consensus exploit the partially synchronous nature of the network to synchronize processes after GST. For that, they use features that are not communication-closed, i.e., they cannot be implemented directly in a round-based model, e.g., a process reacts to messages sent in rounds different from the process's current round. The key to ensure synchronization is to fine-tune the moment when processes locally switch rounds, such that after GST all correct processes are roughly in the same round. The number of synchronized correct processes is determined by the system being tolerant to byzantine or benign faults. Byzantine systems are parametrized by the number of tolerated byzantine processes, denoted $F$.

Instead of using a distributed clock to define the round switch, like in [Dwork et al. 1988], we use hardware clocks and we introduce two synchronization primitives that trigger the termination of the message accumulator:

Catch-up. The message accumulator terminates and the process switches to a future round $r$, when it receives $F+1$ messages sent in rounds greater than or equal to $r$, where $F$ is a parameter of the system, the maximal number of byzantine processes.

Synchronize $F+1$ processes. The message accumulator terminates and the process switch to the next round only if it observes that at least $F+1$ correct processes switched to the next round, where $F$ is again the number of byzantine processes.

Each round is parametrized by the enabling/disabling of these primitives for protocol-specific values of $F$. To compute a round switch, the runtime of RESYNC integrates the implementation of these primitives (when enabled) with the timeouts (or other conditions) defined in the message accumulator. We show that the execution model implemented by the runtime of RESYNC matches the theoretical guarantees for eventual synchrony provided in [Dwork et al. 1988], under similar assumptions. 
Evaluation. We evaluated ReSync on one benign and one byzantine implementation of Paxos and compared it against LiBPAxos3 [Sciascia 2016], an open source implementation of the Paxos algorithm, ETCD an implementation of consensus based on Raft [Ongaro and Ousterhout 2014], and BFT-SMARt [Bessani et al. 2014], an open source implementation of byzantine agreement. RESYNC performance are at most $30 \%$ worse than LiBPAxos3 for a small number of replica, and $10 \%$ faster with more replicas. The results are show a $3.5 \times$ improvement of RESYNC over PSYNC and RESynC and $25 \times$ faster than GooLONG [von Gleissenthall et al. 2019].

Contributions. We propose a new programming abstraction, called ReSync, embedded into the ScAla programming language. We are the first to propose a language that lies between the synchronous and the asynchronous paradigms. Our main contributions are:

- We propose a new round structure that lets programmers use asynchronous optimizations without breaking the synchronous structure.

- RESYNC is suitable for both benign and byzantine fault-tolerant protocols.

- RESync allows the algorithm designer to write custom code that locally controls the round boundaries, i.e., the message accumulator with synchronization primitives, expressing a wide range of protocols.

- RESYnC compiles to efficient asynchronous code, that can be executed over any asynchronous network. The runtime environment ensures a sound and efficient round structure for benign and byzantine faults, based on seminal work by Dwork et al. [1988].

\section{OVERVIEW}

A program in RESYNC is parametric in $N$, the number of processes, and $F$, the number of byzantine ones among them. All (correct) processes execute the same code. A program is a sequence of rounds, called phase. In a round, processes send and receive messages, and then update their local state (depending on the set of received messages). RESync has a round-based semantics, which facilitates writing programs, and an equivalent runtime asynchronous semantics to execute programs efficiently. In the following, we focus on the round-based semantics of RESYNC, where rounds proceed in lockstep. Thus, there are no interleaving of actions performed by processes in different rounds.

Example. As example protocol, we consider a round-based version of ViewChange [Milosevic et al. 2009], a protocol used in PBFT [Castro and Liskov 2002]. The goal of PBFT is to receive requests from a client, and to store all of them on $N$ replicas, in the same order, tolerating $F$ byzantine faulty processes, where $N=3 F+1$. Ideally, all replicas have the same history of client requests, however due to faults this is not the case. ViewChange ensures that a majority of correct replicas-i.e., at least $F+1$ correct replicas-agree on the most recent history of requests. To this, it elects a leader, which makes sure that all the replicas in its quorum agree on the most recent history.

The protocol is structured in three rounds, which form a phase. Fig. 1 shows an execution of ViewChange. In the first round, called DoVC, all replicas broadcast their current history (allto-all communication). We assume authenticated communication channels between any two processes [Milosevic et al. 2009; Srikanth and Toueg 1987], i.e., the receiver of a message

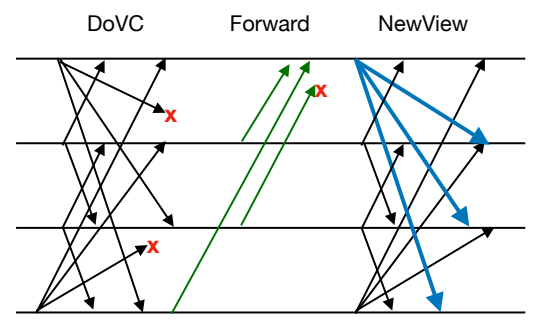

Fig. 1. Lock-step executions for $N=4$ and $F=1$. 
can be sure which process sent the message. All processes, including the leader, wait for $2 F+1$ messages. However, only $F+1$ of these messages may come from correct replicas, which does not provide sufficient information to compute the most recent history of the system. The leader's ID is a function of the phase number (rotating leader), so the protocol uses the same leader in the next two rounds.

In byzantine systems, receiving one message is not sufficient to trust the received value, because byzantine processes might send different (possibly faulty) values to different processes. Therefore, to help the leader trust the received histories, in the second round, called Forward, the replicas that receive at least $2 F+1$ messages in the first round, forward these messages to the leader. The leader trusts an history $h_{i}$ sent by process $p_{i}$, if $F+1$ processes acknowledge that they received $h_{i}$ from $p_{i}$ If the leader receives $2 F+1$ trusted values, based on them it computes, in the second round, the most recent trusted history in the system.

In the third round, called NewView, the leader broadcasts the previously computed history and the set of trusted pairs that the computation was based on. Also in the third round, because the leader might be byzantine, the other processes echo to the entire network the acknowledgments sent to the leader (in the second round). A process $p$ that receives the leader's message, checks that the latest history was correctly computed, using the messages from the other replicas to trust the values communicated by the leader.

The protocol executes these three rounds in a loop, because the execution of one phase does not guarantee that a majority of correct replicas are up to date, e.g., if the leader is byzantine. Fig. 2 shows an execution of a phase where although the leader is not byzantine, there are too many faults in the system to update sufficiently many replicas.

The algorithm is safe if less than a third of the processes are faulty and it is live, if eventually there is a good period where a quorum can

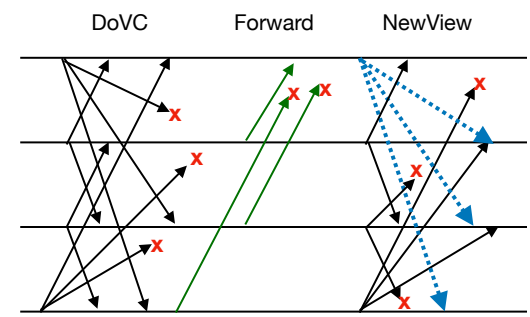

Fig. 2. Lock-step executions for $N=4$ and $F=1$. communicate within itself and with the leader. This is an instance of partial synchrony, which states that eventually all correct processes can talk to each other.

Encoding in RESYNC. The code of the first round, i.e., DoVC, is given in Fig. 3. The round implements four methods: send, defining the messages to be sent, receiveInit and receive computes the set of messages received in the round, i.e., the message accumulator, and finishRound that defines the round computation based on the received messages. In this round, all processes broadcast their current histories. Therefore, send uses the primitive broadcast and returns, at line 7 , a (key,value) map, where the keys are all the process identities (the recipients) and the value is the history of the sender, i.e., a sequence of requests (type Hist). The method send is executed synchronously by all processes.

Message accumulator. In classic round-based models [Santoro and Widmayer 2007], the set of received messages is a subset of the sent messages, non-deterministically chosen by an adversarial environment. We enhance the round-based model with a message accumulator (specific to each round) that receives messages one by one. This gives the programmer the capabilities to react to a non-deterministic execution environment. The message accumulator is defined by a Progress object and two methods that use it, receiveInit (line 9) and receive (line 14). The main bottleneck of round-based models is an inefficient round-switch policy that requires synchronizing 


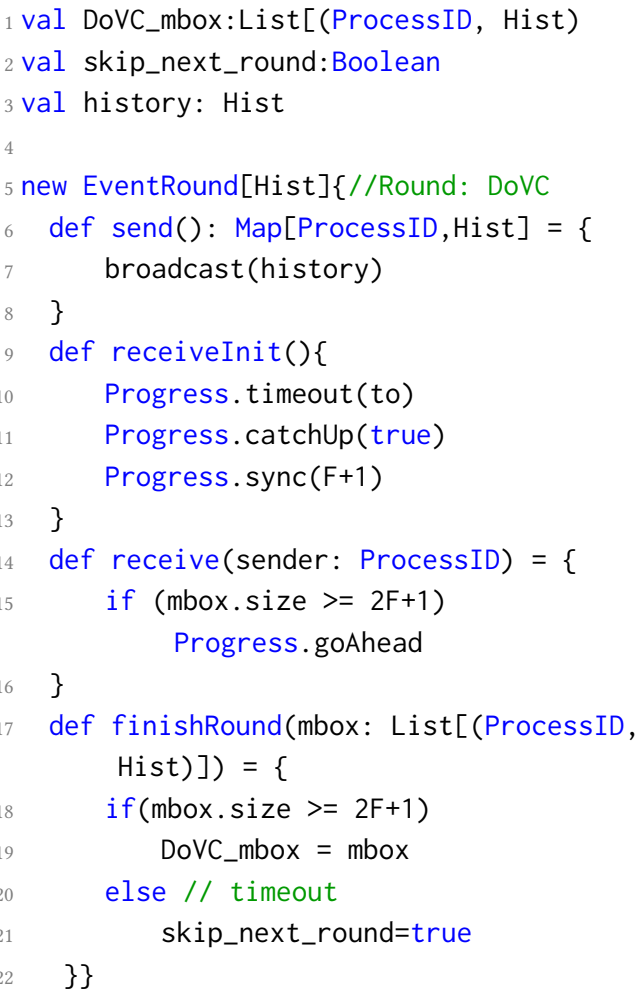

Fig. 3. The 1st round of ViewChange (PBFT) in ReSync.

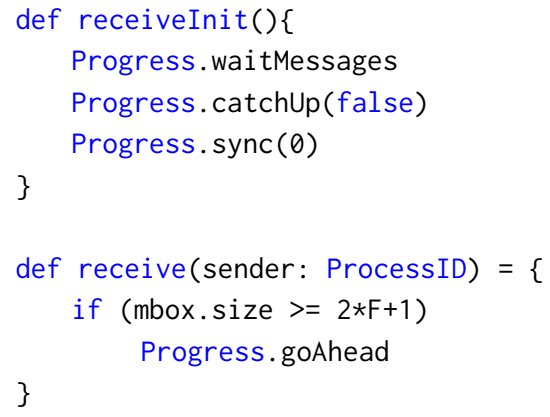

Fig. 4. Another message accumulator for the round in Fig. 3

correct process. Using the methods of Progress, called progress conditions, the programmer guides the execution platform of RESYNC, speeding up or slowing down the default round-switch policy. They are high-level constructs implemented by the execution platform. For example, using Progress.timeout (to) the programmer fixes in line 10 the time interval the execution engine waits for the messages of the current round, i.e., it waits for to milliseconds. Two other progress conditions are enabled.

Table 1. Network assumptions.

\begin{tabular}{|c|lc|}
\hline & Lossy links & Reliable link \\
\hline benign process crash & $F=0$ and UDP & $F=0$ and TCP \\
authenticated byzantine processes & $F>0$ and UDP & $F>0$ and TCP \\
\hline
\end{tabular}

A message accumulator is designed taking into account the underling network. In this paper we consider different fault models, given in Table. 1. Regarding process failure, processes can crash (and not recover) or they can be byzantine. A byzantine process does not follow the protocol, it may send any messages except forging the identity of other processes: We assume authenticated byzantine processes which cannot impersonate other processes, and this is done using digital signatures. The number of byzantine processes, denoted $F$, is a parameter of the program. If $F$ equals zero then the program tolerates only benign faults. The size of the network is fixed by the parameter $N$. For the 
link failures, we consider either lossy links, where messages can be lost, reordered, duplicated, or reliable links where every message sent is eventually received. In practice lossy links are ensured by UDP and reliable links by TCP. The design of a custom message accumulator can take advantage of any other network properties the programmer is aware of, it is not restricted to the network assumptions discussed here. In this paper, we present a run-time algorithm in Section 5 that can be parameterized and works for each of the four options from Table. 1. The current implementation in Section 6 only considers the reliable communication cases.

Synchronization primitives. ReSync has two synchronization primitives, Progress . catchUp and Progress.sync. They are implemented by the execution platform, for the types of network given in Table. 1. Note that a network with lossy links is a weaker network assumption than reliable links. These primitives do not have a round-based definition for lossy links (Progress . catchUp does not have a round-based implementation even over networks that ensure reliable links).

The primitive Progress . catchUp(true), Fig. 3 line 11, enables a process to switch rounds faster at runtime, as soon as it has received $F+1$ messages from different processes with a higher round. It is used to resynchronize processes by advancing a slow process to the round of a faster correct one.

The primitive Progress. sync $(\mathrm{F}+1)$, Fig. 3 line 12 requires that $F+1$ correct processes switch rounds together, at runtime. When sync is used a process that is in round $\mathrm{cr}$ moves to the next round $c r+1$ when it receives at least $2 F+1$ messages from different processes for the current round $c r$, or a higher one. Note that the network might drop more than $F$ messages in a round (we recall $N=3 F+1$ ). If the timeout expired and less than $2 F+1$ messages are received, the execution platform first increases the timeout but if this is not sufficient, it resends the messages of the current round (if the round does not use an all-to-all communication, the execution platform inserts dummy messages to implement the synchronization requirement). ${ }^{1}$ Progress. sync $(F+1)$ overrules the timeout constraints set by Progress. timeout (to). Byzantine system cannot resynchronize (after an asynchronous period under partial synchrony) unless at least $F+1$ processes are within the same round [Dwork et al. 1988]. Therefore, even if Progress. sync $(F+1)$ potentially slows down the round switch, it is necessary for byzantine consensus protocols.

For each round, the Progress object is configured in the method receiveInit. Messages are received one by one: the method receive takes as input a received message and implements the conditions under which this should be the last message received in the current round. Each process, in each round, has a fresh mailbox, that contains the queue of messages received (inputs to receive) by the process in that round. This mailbox is populated by the execution platform. The execution of the message accumulator starts with receiveInit, continues with multiple calls to receive, and stops when the progress conditions are met. The accumulator in Fig. 3 stops either due to Progress. catchUp or because Process. sync $(F+1)$ holds and either the timeout expired or the control reached Progress.goAhead in Fig. 3 line 15. Since Process.sync $(F+1)$ is enabled, the message accumulator continues to collect messages until the progress condition is met even if the timeout expired. The messages accumulators of the same round execute asynchronously across processes.

Round computation. The method finishRound implements the computation done by a process in a round, based on the set of received messages. All (non-byzantine) processes execute the same code, however due to different mailboxes and branching, processes have different behaviors finishRound is executed when a process stops receiving messages. The only computation done in the first round in ViewChange is to store the mailbox in the variable DoVC_mbox, in line 19. Across

${ }^{1}$ The implementation of Progress. sync $(F+1)$ is possible only for partially synchronous networks. 
rounds, RESync respects the classic round-based semantics: at the end of a round the mailbox of each process is purged and the computation moves in lockstep to the next round.

Different message accumulators. To solve byzantine consensus, it is sufficient that only some rounds force $F+1$ correct processes to be synchronized. Therefore, in ViewChange at least one of the three rounds should enable Process . sync, but not necessarily the first one. If we consider the message accumulator from Fig. 3 with Process. $\operatorname{sync}(\theta)$, then the message accumulator might stop due to an expired timeout, before receiving $2 F+1$ messages. Therefore when finishRound is executed, line 21 is reachable and skip_next_round is true. In ViewChange, processes that do not receive $2 F+1$ messages in the first round move on to the next phase. In the round-based structure, processes cannot skip rounds. However, they are not forced to send messages or do any computation in a round. The flag skip_next_round is used to encode the behavior of these processes, which go through the second and third round of the current phase without sending any messages or doing any computation.

Let us consider the message accumulator in Fig. 4 which uses Progress. wai tMessages instead of Progress.timeout. The semantics of Progress.waitMessages states that calls to receive are made until Progress. goAhead is reached. Since Progress. catchUp is disabled, the only way for a process to switch round is if $2 F+1$ messages are received. This message accumulator can get stuck, if the program is executed over a network that provides unreliable communication. However, if executed with reliable communication between correct processes, e.g., TCP, it is an implementation (in the new round model) of the synchronization primitive Process . sync $(F+1)$.

The message accumulator can also depend on the value in the payload of messages. Let us look at the two-phase commit protocol. Fig. 5 presents the first two rounds - i.e., the first phase - of the protocol. It is an atomic commitment protocol, executed by $n$ benign processes (that is all processes follow the protocol). This protocol assumes crash recovery, i.e., the nodes have stable storage and they can resume operation after a crash. Therefore, the processes block until they receive the messages they expect.

In the example, the transaction identifier is an integer that the leader proposes (at line 5) to all processes. Processes blocks until they receive the message from the leader (lines 9 and 13). Each process checks locally whether it can locally commit the transaction (without violating the consistency of the database) at line 16. In the second round, on the right, processes send (at line 4) their vote to the leader. The two-phase commit protocols says that a transaction can commit only if all the participants agree. Therefore, the leader waits for $N$ "yes" votes or a single "no" vote (line 11). In the next two rounds, which we omit for brevity, the coordinator sends a commit or abort message to all and after it receives acknowledgments from all processes, it informs the client of the decision and restarts with a fresh transaction.

In our example, we do not give concrete values for timeouts. The actual timeout value might vary across rounds as they are influenced by the amount of computation required by handling messages. For example, if we consider networks with non-authenticated communication channels, messages are signed using public encryption policies, and the signatures are checked by the recipients. The timeout depends on the size of the information to be signed and on the signature algorithm. By choosing a timeout that takes only the network delay into account, the round switch may happen too fast, and processes could miss messages that are delivered by the network.

Runtime executions. Fig. 6(a) and Fig. 6(b) show two runtime executions of ViewChange in RESYnc. At runtime, processes are not all in the same round (due to the asynchronous nature of the network). Therefore, processes receive messages from different rounds out of order. The runtime discards the late messages and stores the messages from the future rounds. It delivers only the messages for the current round. Since Progress. sync $(F+1)$, at runtime, a process will not finish 

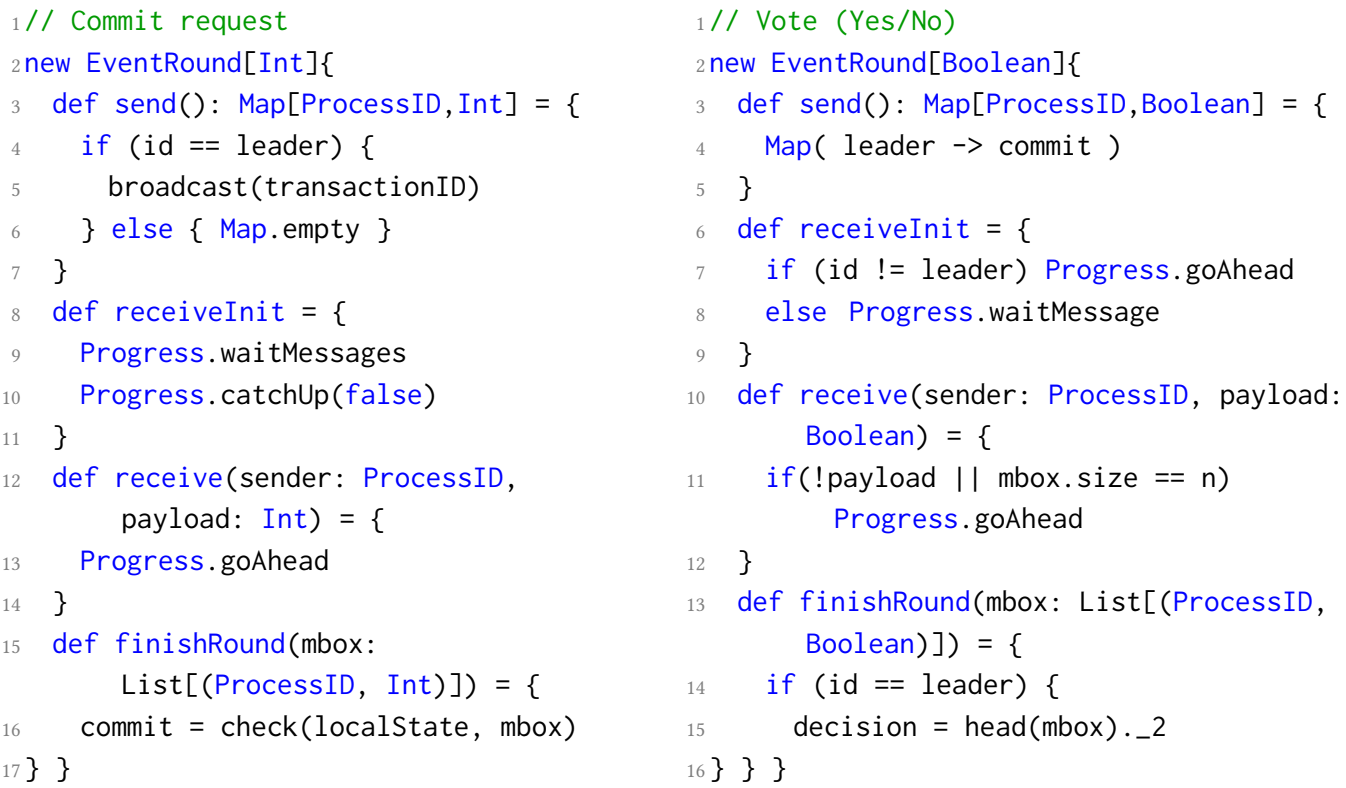

Fig. 5. Voting phase of the Two phase commit protocol in RESYNC.

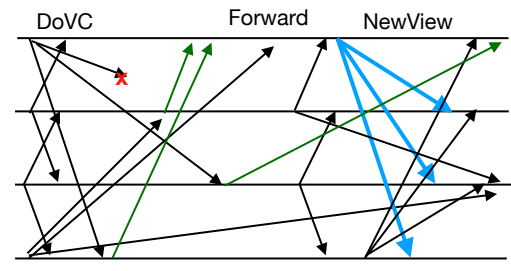

(a)

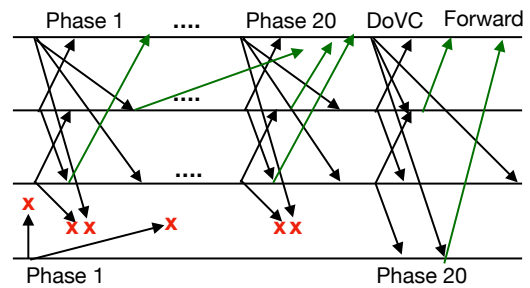

(b)

Fig. 6. Runtime executions for $N=4$ and $F=1$.

DoVc when the timeout expired, unless it has received at least $2 F+1$ messages for the current round (or a higher one). However, a process finishes the other two rounds independently of the number of received messages.

Not all processes are forced to wait for $2 F+1$ messages. The progress condition catchUp makes processes jump to a future round, if they received more than $F+1$ messages for that round (or a higher one). For example, in Fig. 6(b), the bottom process is slowed down in the round DoVc of the first phase and jumps to round DoVC of the 20th phase, when it receives $F+1$ messages for it. This process is not forced to wait for $2 F+1$ messages as the reception of $F+1$ messages for a higher round guarantees that there were (other) $F+1$ correct processes synchronized. We show that the runtime executions are refinements of the lockstep executions: processes at runtime go through the same sequence of local states as in the round-based semantics. For example, the execution in Fig 6(a) is a refinement of the execution in Fig 1. 


\begin{tabular}{|c|c|c|c|}
\hline program & $::=$ & $\begin{array}{l}\text { interface } \\
\text { body }\end{array}$ & $\begin{array}{l}\text { EventRound }_{\mathrm{M}} \\
\quad \text { mbox: List }[P \times \mathrm{M}]\end{array}$ \\
\hline \multirow[t]{4}{*}{ body } & $::=$ & var_decl* & Progress: ProgressC \\
\hline & & pure_fun* & send: ()$\rightarrow[P \mapsto \mathrm{M}]$ \\
\hline & & init & receiveInit: ()$\rightarrow()$ \\
\hline & & phase & receive: $(s: P, m: \mathrm{M}) \rightarrow())$ \\
\hline phase & $::=$ & round $^{+}$ & finishRound: $(\operatorname{mbox}) \rightarrow()$ \\
\hline
\end{tabular}

Fig. 7. ReSync abstract syntax.

\section{SYNTAX OF RESYNC}

The syntax of an RESynC program is given in Fig. 7. A program defines the code executed by one process. In RESYNC all processes execute the same code. However, within a round the programmer can encode different roles (e.g., coordinator, follower) by branching. The branching conditions may be over the process identifier or received messages (which can vary non-deterministically across processes).

A program is defined by an interface and the program's body. The interface is an object with a list of methods that are used to communicate with other distributed programs. The interface has inbound operations, for example to get a new client request, and outbound operations (callbacks), called to deliver some results to the client, e.g., acknowledgments that the request has been processed. The program's body has variables declaration, an initialization function, the main distributed computation part, and sequential pure function. All variables are local to a process. The communication between processes is done exclusively by message passing.

Variables. All ReSync programs have several predefined variables: $N$ the total number of processes, $F$ the number of byzantine processes (both parameters of the program), and $r$ the round number. These variables are read-only.

Initialization. Each program implements an init method, which configures the initial state of a process: the initial values are obtained using the inbound interface operations. The method init takes as input interface objects, it does not return any values. It modifies the process's variables without sending or receiving messages. Implicitly, init initializes the round number $r$ to zero.

Rounds. The distributed computation part is structured into a fixed sequence of rounds, called phase. Each round is an instance of an abstract class EventRound, whose structure is described in Fig. 7. Rounds are parameterized by a payload type $M$ and have two attributes: 1 ) mbox, of type $\operatorname{List}[(P, M)]$, is a queue of messages, where $P$ is the process type, and a progress object, Progress, where ProgressC is the class of progress conditions. In the code snippets, we use process identifier (ProcessID) for $P$. For the semantics, these two types are the same. Rounds contain a few methods which define their behaviors. The methods send and finishRound must be defined by the program. An implementation of the methods receiveInit and receive is optional. If receiveInit and receive are implemented, the round is called an open round, otherwise it is called a closed round. In closed round Progress. catchUp and Progress. sync $(F+1)$ are enabled by default.

The type of messages exchanged in different rounds might differ, however within a round, all messages have the same payload type. The control flow of a round is a sequence of method calls, starting with send, followed by receiveInit and a loop of calls to receive, if the latter two are present, and lastly finishRound. The round variables mbox and Progress are visible in all methods of the same round. 
Sending messages. The method send does not take any inputs, and returns a partial map from process identity to a payload type, i.e., $[P \mapsto \mathrm{M}]$, which associates receivers with payload values, i.e., the sent messages. The method send is side-effect free w.r.t. the algorithm variables.

Message accumulator. A message accumulator collects the messages delivered by the network in a round (to the process that executes it). The message accumulator stops, when a certain condition holds. Next, the method finishRound is executed and the control switches to the next round.

The algorithm designer implements the message accumulator by defining receiveInit and receive and controls when the accumulator terminates using different progress conditions. A progress condition is a method or an attribute of a ProgressC object. They impose different conditions on when the accumulator may stop receiving messages:

- Progress.timeout(to: Milliseconds), the accumulator is enabled to end receiving messages after a timeout expired, that is to time units after the round started;

- Progress.goAhead, the accumulator is enabled to end as soon as the method goAhead is called;

- Progress.waitMessages is a flag; when set, it means that there is no timeout to stop the message reception.

- Progress.catchUp(b: Boolean) allows or disallows the execution platform to (re-) synchronize processes under a partial synchrony assumption;

- Progress.sync(k: Int) is a global synchronization primitive that constraints the message accumulators of $k$ correct processes to end synchronously.

The method receiveInit configures the progress conditions. The programmer may implement different progress conditions for different processes in the same round by branching. The method receive defines the mbox and checks if the message accumulator should terminate. It takes as input an incoming message, that is, a pair (sender,value), where sender is the process which sent the message, and value a value of the round's the payload type. Each execution of receive adds the input pair (sender, value) to the mbox of the current round. In receive the programmer uses Progress. goAhead to terminate the message accumulator when a condition on the mailbox computed so far holds.

When the methods receiveInit and receive are not implemented, the mailbox is a nondeterministic subset of the sent messages.

Round computation. In the method finishRound the programmer defines how the process state changes at the end of a round. The method finishRound takes as input the queue of messages received, i.e., takes as input mbox. It may call outbound operations from the interface, to communicate the results of the computation to a client.

\section{LOCK-STEP SEMANTICS OF RESYNC}

Given a program $\mathcal{P}$, all (non-byzantine) process execute, in a loop, the sequence of rounds defined in the program. The semantics of a program is given by the synchronized parallel composition of the transition systems associated with each process. We denoted by $[[\mathcal{P}]$ the set of executions defined by the transition system in Fig. 8, Fig. 9, Fig. 10, and Fig. 11.

The execution progresses in lockstep, that is, all processes execute the same round in the same time. Within a round, the methods send and finishRound are executed synchronously by all processes, while the executions of receiveInit, receive, and the network transitions are interleaved across processes within the round boundaries. Locally, within a round, a process executes a sequence of calls to methods corresponding to

$$
\text { init; } \left.\left(\text { send } ;\left(\text { receiveInit } ; \text { receive }{ }^{*}\right) \mid \text { NondetNet }\right) ; \text { finishRound }\right)^{*}
$$


where NondetNet represents a network transition. All processes execute the same sequences of rounds.

The state $S$ of a RESync program over the set of processes $P$ is represented by the tuple $\langle$ gstep, $s, r, m s g\rangle$ where:

- gstep $\in\{$ Send, Recv, Net, Fin $\}$ indicates whether the next method is send, receive, a network transition, or finishRound, respectively;

- $s \in[P \rightarrow$ Vars $\cup\{$ lstep $\} \rightarrow \mathcal{D}]$ stores the local states of the processes, Vars is the union of all variables, $\mathcal{D}$ is the domain of values, and lstep is a special variable taking a value in $\{$ Send, Recv, Net, Fin $\}$ and ;

- $r \in \mathbb{N}$ is a counter for the executed rounds;

- $s m s \subseteq 2^{P, M, P}$ is a set of sent messages in a round and $d m s \subseteq 2^{P, M, P}$ is a ordered set of delivered messages, all the messages in sms and $d m s$ have a different pair of sender/receiver, i.e., a process can only send one message to another process in each round.

To model correct, crashed, and byzantine processes we use the sets $C o P, C r$, and ByzP. CoP is the set of correct processes, $\mathrm{Cr}$ is the set of crashed processes, and ByzP contains the byzantine processes. These sets form a partition of the processes, i.e., $P=C o P \uplus C r \uplus B y z P$. $N$ and $F$ take the values $N=|P|$ and $F=|B y z P|$. For crashed processes, we set their local state to the special $\perp$ value, i.e., for a store $s$ we have $C r=\{p \in P \mid s(p)=\perp\}$. Crashed processes never recover. $\operatorname{Pr}$ is a shorthand the set of running processes, i.e., $\mathrm{Pr}=\mathrm{P} \backslash \mathrm{Cr}$.

The rules for the round structure where the processes work in lockstep are shown in Fig. 8. Later, we will see additional rules for the network (Fig. 9), closed rounds (Fig. 10), and open rounds (Fig. 11). Global lockstep transitions are written as $S \stackrel{E, I, O}{\Longrightarrow} S^{\prime}$ where $S, S^{\prime}$ are states, $E$ is a set of labels that identify network transitions, $O$ is a set of labels defined from the names of the methods in the interface. $I$ is a set of labels of internal transitions (not in the interface. A client of a RESyNC program can only observe the transitions with labels in $O$. Local transitions of a process are written with $\longrightarrow$ and transition of the network are denoed with $\longmapsto$.

Init. Initially the state of the system is undefined, denoted by $*$. The first transition of the system, captured by the rule INIT in Fig. 8, defines the initial state of the protocol by calling init on all processes. All processes execute init in lockstep (synchronously). The method init does not return a value and defines the local state of a process using inbound operations from the interface. The global transition INIT emphasizes the inbound operations called by init, denoted $o_{p}$, where $o$ is the operation's name and $p$ is the process executing it. INIT sets the round counter to 0 , the message pools are empty (there are no messages in the system), and globally the system transitions to a Send state, i.e., gstep = Send . Rounds are executed in a loop. Next, we define the semantics of a round.

Send. In every round, the first transition is a global one, which executes locally in lockstep the method send on all processes. Its semantics is formalized by the rules Send-Open and Send-Closed in Fig. 8. Only one of the two rules applies in a round. Send-Open is applied when init and receive are defined. The control label gstep becomes Recv, i.e., the control goes to the implementation of the round's message accumulator. Send-Closed is applied when init and receive have the default implementation and in this case the control goes to the network, that is gstep equals Net, otherwise gstep equals Recv. The method send has no side effects at a process and returns a map from receivers to payload values, i.e., the messages sent by the process that executes send. The rules Send-Closed, resp. Send-Open add the messages sent by processes to the pool of sent messages, sms. The messages in sms are triples of the form (sender, payload, recipient), where the sender and 


$$
\begin{aligned}
& \text { INIT } \\
& \frac{\forall p \in P r . * \stackrel{\operatorname{init}(), o_{p}}{\longrightarrow} s(p) \quad O=\left\{o_{p} \mid p \in \operatorname{Pr}\right\}}{*,\{\text { init } p() \mid p \in \operatorname{Pr}\}, O} \underset{ }{\Longrightarrow}\langle\text { Send, } s, 0, \emptyset, \emptyset\rangle
\end{aligned}
$$
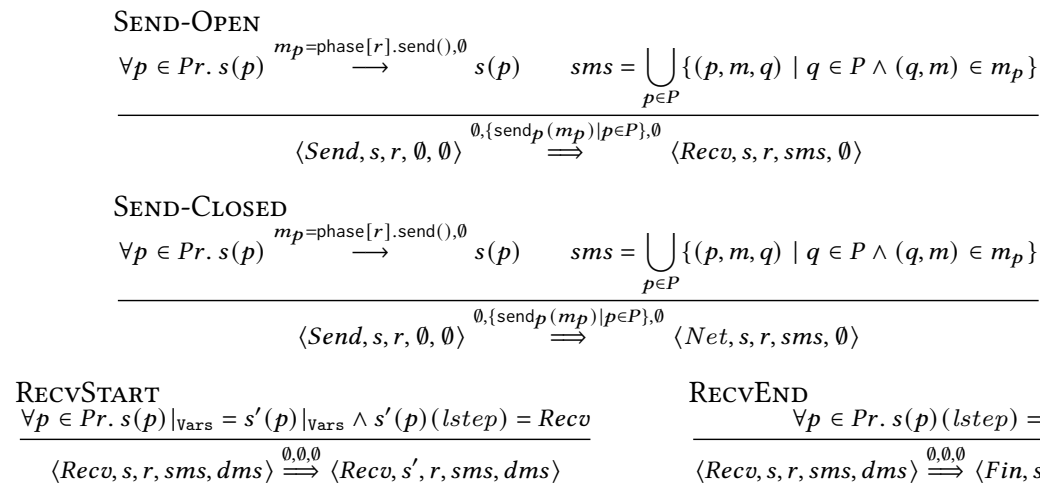

$$
\begin{aligned}
& \text { RecvEnd } \\
& \frac{\underset{\operatorname{RECVEND}}{\forall p \in \operatorname{Pr} . s(p)(\text { lstep })=\text { Fin }}}{\langle\text { Recv, } s, r, s m s, d m s\rangle \stackrel{\emptyset, \emptyset, \emptyset}{\Longrightarrow}\langle\text { Fin }, s, r, s m s, d m s\rangle}
\end{aligned}
$$

FinishRound

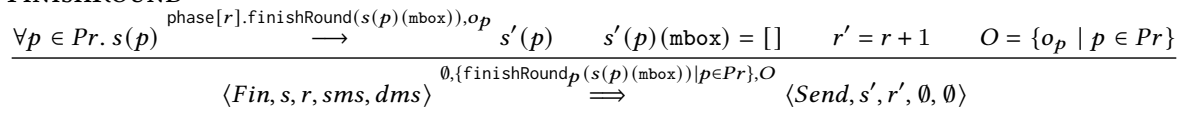

Fig. 8. The global semantics of ReSYNc. A transition $s(p) \stackrel{\text { phase }[r] . m, o}{\rightarrow} s^{\prime}(p)$ denotes that $p$ executes, in local state $s(p)$, the method $m$ of the EventRound phase $[r]$. The execution produces observable events $o$, corresponding to calls to methods from the interface.

$$
\begin{aligned}
& \frac{\text { DroP } s m s=s m s^{\prime} \uplus\{m\}}{\langle s, s m s, d m s\rangle \stackrel{D r}{\longmapsto}\left\langle s, s m s^{\prime}, d m s\right\rangle} \\
& \begin{array}{l}
\text { DELIVER } \\
s m s=s m s^{\prime} \uplus\{(p, v, q)\} \quad\left(p \in B y z P \vee v=v^{\prime}\right) \quad d m s^{\prime}=d m s \cup\left\{\left(p, v^{\prime}, q\right)\right\}
\end{array} \\
& \frac{s^{\prime}(p)=\perp \quad \forall q . q \neq p \Rightarrow s(q)=s^{\prime}(q)}{\langle s, s m s, d m s\rangle \stackrel{C r}{\longmapsto}\left\langle s^{\prime}, s m s, d m s\right\rangle} \\
& \langle s, s m s, d m s\rangle \stackrel{D v}{\longmapsto}\left\langle s, s m s^{\prime}, d m s^{\prime}\right\rangle
\end{aligned}
$$

Fig. 9. Network transition on the message pools.

$$
\begin{aligned}
& \text { Closed-NetworkSteps } \\
& \frac{\langle s, s m s, d m s\rangle \stackrel{N}{\longmapsto}\left\langle s^{\prime}, s m s^{\prime}, d m s^{\prime}\right\rangle}{\langle N e t, s, r, s m s, d m s\rangle \stackrel{N, \emptyset, \emptyset}{\Longrightarrow}\left\langle N e t, s^{\prime}, r, s m s^{\prime}, d m s^{\prime}\right\rangle} \\
& \text { Closed-Mailbox } \\
& \forall p \in \operatorname{Pr} . s(p)(\text { lstep })=N e t \wedge s^{\prime}(p)(\text { lstep })=\text { Fin } \\
& \frac{\left.\wedge s(p)\right|_{\text {Vars }}=\left.s^{\prime}(p)\right|_{\text {Vars }} \wedge s^{\prime}(p)(\operatorname{mbox})=[(q, m) \mid(q, m, p) \in d m s]}{\langle\text { Net, } s, r, s m s, d m s\rangle \stackrel{\emptyset, \emptyset, \emptyset}{\Longrightarrow}\left\langle\text { Fin, } s^{\prime}, r, s m s, d m s\right\rangle}
\end{aligned}
$$

Fig. 10. Network transitions for closed rounds.

receiver are processes and the payload has type $\mathrm{M}$. The triples are obtained from the map returned by send to which we add the identity of the process that executed send. 


\section{Progress transitions}
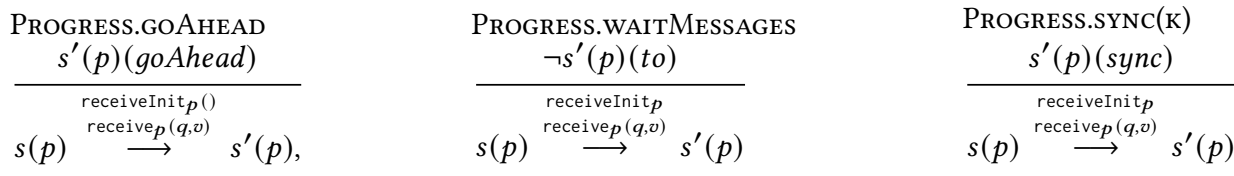

Progress.catchUp(TR
$s^{\prime}(p)($ catch $U p)$
$s(p) \stackrel{\text { receiveInit } p}{\text { receive } p_{p}(q, v)} s^{\prime}(p)$

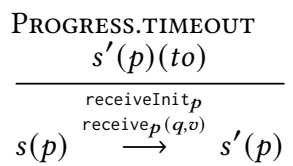

\section{Accumulator transitions}

Acc-Init
$\frac{s(p)(\text { lstep })=\text { Recv }\left.\quad s(p) \stackrel{\text { receiveInit }}{\longrightarrow} s^{\prime}(p) \quad s(p)\right|_{\text {vars }}=\left.s^{\prime}(p)\right|_{\text {vars }}}{\langle s(p), s m s, d m s\rangle \stackrel{\{\text { receiveInit }}{\longrightarrow}\}, \emptyset}\left\langle s^{\prime}(p), s m s, d m s\right\rangle$

AcC-Recv

$$
\frac{\begin{array}{ccc}
\neg s(p)(\text { goAhead }) & d m s=d m s^{\prime} \uplus\{(q, v, p)\} & s(p) \stackrel{\text { receive }_{q}(q, v)}{\longrightarrow} s^{\prime}(p) \\
s(p)(\text { lstep })=s^{\prime}(p)(\text { lstep })=\left.\operatorname{Recv} \quad s(p)\right|_{\operatorname{Vars}}=\left.s^{\prime}(p)\right|_{\operatorname{Vars}} & \left.s^{\prime}(p)(\operatorname{mbox})=(q, v):: s(p) \text { (mbox }\right)
\end{array}}{\langle s(p), s m s, d m s\rangle \stackrel{\text { receive } \left._{p}(q, v)\right\}, \emptyset}{\longrightarrow}\left\langle s^{\prime}(p), s m s, d m s^{\prime}\right\rangle}
$$

Acc-End

$$
\begin{aligned}
& s(p)(\text { lstep })=\operatorname{Recv} \quad(s(p)(\text { GoAhead }) \vee s(p)(\text { to }) \vee s(p)(\text { catch } U p)) \\
& \underline{\left.s(p)\right|_{\text {Vars }}=\left.s^{\prime}(p)\right|_{\text {Vars }} \quad s^{\prime}(p)=\text { Fin } \quad \neg s^{\prime}(p)(\text { GoAhead }) \quad \neg s^{\prime}(p)(\text { to })} \\
& \langle s(p), s m s, d m s\rangle \stackrel{\emptyset, \emptyset}{\longrightarrow}\left\langle s^{\prime}(p), s m s, d m s\right\rangle
\end{aligned}
$$

\section{Global transitions}

ACC-NeTWORKSteP

$\frac{\langle s, s m s, d m s\rangle \stackrel{N}{\longmapsto}\left\langle s^{\prime}, s m s^{\prime}, d m s^{\prime}\right\rangle}{\langle\operatorname{Recv}, s, r, s m s, d m s\rangle \stackrel{N, \emptyset, \emptyset}{\Longrightarrow}\left\langle R e c v, s^{\prime}, r, s m s^{\prime}, d m s^{\prime}\right\rangle}$
Acc-ProcessStep

$\frac{\langle s, s m s, d m s\rangle \stackrel{I, \emptyset}{\longrightarrow}\left\langle s^{\prime}, s m s^{\prime}, d m s^{\prime}\right\rangle}{\langle R e c v, s, r, s m s, d m s\rangle \stackrel{\emptyset, I, \emptyset}{\Longrightarrow}\left\langle R e c v, s^{\prime}, r, s m s^{\prime}, d m s^{\prime}\right\rangle}$

Fig. 11. Message accumulator transitions.

After sending messages the control passes to the network that non-deterministically decides which messages to deliver and which to alter.

Network transitions. The pool of messages sms and the lists of delivered messages $d m s$ are managed by a special network process that modifies the pool of messages sms and $d m s$ (for simplicity the rules omit its identity), and in the case of open rounds controls also the progress conditions. The rule Deliver, in Fig. 9, captures how the network takes a message $m$ from the message pool sms and puts it into the delivery set $d m s$. Messages sent by Byzantine faulty processes can be altered before being delivered. Thus we follow the modelling approach of communication faults [Biely et al. 2007; Santoro and Widmayer 1989]. This way of modeling means we can keep uniform rules for sending and processing messages. The rule Drop captures how the network can drop sent messages by 
removing them from the send pool sms. Processes may crash (see Crash); once crashed, processes never recover.

Message reception in closed rounds. When receiveInit and receive are missing (they are not redefined), after Send-Closed, the rule NoRecv-NetworkSteps is applied, a nondeterministic number of times, followed by the rule NoRecv-Mailbox, from Fig. 10. The mailbox of each process is set to be equal with the content of the delivery queue of the process. The mailbox is totally under the control of the network, that populates mbox with a non-deterministic chosen (possibly altered) subset of the sent messages.

Message accumulator (message reception in open rounds). The implementation of the message accumulator starts after sending messages (after apllying SEND-OPEN), by updating the local variable lstep to Recv (see rule RecvStart in Fig. 8). To compute the mailbox of an open round, process executes first receiveInit (see rule Acc-Init in Fig. 11) and afterwards a loop of calls to receive (see rule Acc-Recv in Fig. 11). Calls to receive stop when the progress conditions configured in receiveInit are met (see rule Acc-End).

Receivelnit. The progress conditions of a round are set in receiveInit. To formally define them we use auxiliary boolean local variables: goAhead, catchUp, to, and sync. Because the semantics is given at the lockstep level and the progress conditions are hints to control the runtime algorithms, the progress condition does not have much impact on the semantics. For instance, the timeout is abstracted as a boolean value and the accumulator can non-deterministically continue receiving messages or end.

Receive. The method receive when executed by process $p$, takes as input a delivered message in the set $d m s$ (see rule Acc-Recv) and adds it to the mailbox of the current round. In each round, at most one message is received from any process, i.e., duplicates if present are eliminated. Another call to receive follows only if the progress conditions are not met, see rule Acc-End in Fig. 11. The message accumulator ends because either the catchUp condition holds, or the synchrony condition holds together with either the goAhead or the timeout condition (see rule Acc-End). Note that the catchUp, sync, and timeout progress conditions are in this lockstep semantics under the control of the network, and the protocol assumes the network respects their semantics. The execution platform provides an implementation of these primitives.

The accumulator transitions are interleaved with the network transitions defined in Fig. 9. While collecting messages, the local variable lstep equals Recv until it gets updated to Fin at the end of the message accumulator. Globally the systems transitions into a Fin global state when all non-crashed processes have their local variable lstep equal to Fin, rule RecvEnd in Fig. 8.

Round-based Algorithm transition. In a FinIsHRound transition (Fig. 8), all processes execute synchronously (without any interference) the method finishRound of the current round. Locally, on each process $p$, the set of received messages $\operatorname{mbox}_{p}$ (computed previously) is the input of finishRound. The finishRound operation might produce an observable transition $o_{p}$. At the end of the round, sms and $d m s$ are purged and $r$ is incremented by 1 .

\section{RUNTIME OF RESYNC}

We introduce an execution platform for RESync over asynchronous networks. The runtime executions are refinements of the lockstep executions from Section 4. For closed rounds, if the underlying network is partially synchronous, the runtime eventually synchronizes correct processes, ensuring that they can communicate reliably with each other. 


\subsection{Runtime Semantics}

The runtime algorithm in Listing 1 defines the code executed at runtime by one process. Listing 1 shows an extract from the actual implementation (which has lines of code) that highlights the control structure of the runtime algorithm. Given a RESYNC program $\mathcal{P}$, the set of runtime executions $[[P]]_{R u n}$ is defined by the asynchronous parallel composition of $N$ systems shown in Listing 1 .

The runtime algorithm defines a wrapper for the RESync code. It has the following structure: declaration of the variables that keep track of the timeout, the round number, and the buffer of delivered messages (line 7), a few auxiliary methods (lines 16-30), and a big while loop where every iteration corresponds to the execution of one round (lines 33-60). All processes go through all rounds in order. Each loop iteration is further split into initialization (lines 38-41), send (line 35), message accumulator (lines 43-55), and finishing the round (lines 57-59). The message accumulator is a loop where at each iteration one message is received and processed. The exit of this loop triggers a round switch.

The runtime uses phase, the sequence of len rounds defined in RESYNC, and the variable currentRound holding the current round number. In Fig. 1, phase[currentRound] is the $\mathrm{k}^{\text {th }}$ EventRound defined in the RESync program, where $k$ equals currentRound\%len. The runtime uses the implementations of send, receiveInit, receive, finishRound given in ReSYNC by calling phase[currentRound]. send, etc. Each call is wrapped by checkProgress that implements the semantics of the progress conditions.

Message reception is implemented using the method receiveWithTimeout in line 45 that either returns a message or null if the timeout (given by the RESYNC program) expired. If the progress condition waitMessages is active, receiveWithTimeout is called with an infinite timeout. The messages delivered to a process are from different rounds. For instance, if two processes $p$ and $q$ are in different rounds, and send messages for their current rounds to some process $r, r$ 's message buffer will contain messages from different rounds. The runtime implements a filtering of these messages. To each sent message it adds as metadata the current round number of its sender. When a message is received, the runtime discards it, if it was sent in a round smaller than the current round of the receiver, and it buffers it in the buffer pendingMessages if it is a message that comes from a future round. The mailbox contains messages only from the process's current round. The default behavior of receiveInit is to set a timeout, enable catchUp and enable the synchronization of $F+1$ processes. The default behavior of receive is to add a received message to the mailbox, when it belongs to the current round. Messages in pendingMessages are moved to the mailbox when the process reaches the round they were sent in. The function checkProgress updates the local variables that gate a round switch at line 44. This function is called after the reception of a message (line 52) and in the beginning of every round with the call of receiveInit (line 41) but also on the buffered set of messages for the current round (line 43) received while the system was operating in a lower round.

CatchUp algorithm. This primitive enables a process to jump over over some rounds at runtime, so that it can catch-up with processes that made quicker progress. Catch-up is a mechanism used by many asynchronous systems, e.g, Paxos, ViewStamped, PBFT. Implementing this primitive requires breaking communication-closure which is possible only at execution time.

In the benign case, i.e., $F=0$, when a message $m$ from a future round $f r$ is received, instead of storing it and continuing with the current round, the runtime jumps to the round $f r$ and starts the message accumulator of $f r$ with $m$ in the mailbox. Note that catch-up is useful to synchronize processes (with the faster ones) the under lossy links, because messages of the current round might be lost, but also under reliable links due to message reorder (see Table 1). The implementation 


\section{Listing 1. ReSync Runtime Algorithm}

// Global constants

id: Pid; $N, F: \mathbb{N}$ //own process id, number of processes, malicious processes round: Array[EventRound $\mathbf{M}$ ] //Program

retransmit: $\mathbb{B} / /$ periodically resend messages in the absence of progress

// State shared with the program

Progress: ProgressC

mbox: List[Msg] $=[] / /$ mailbox of the current round

// Internal state

currentRound, nextRound: $\mathbb{N}=0$

roundStart, timeout: Time

strict, inSync: $\mathbb{B}=$ false $/ /$ flags to control the progress

maxRound: Map $[P i d, \mathbb{N}]=\operatorname{Map}[$ for $(p<-\operatorname{Pid})$ yield $(p, 0)] / /$ keep the max round seen for each process pendingMessages: $\operatorname{Map}[\mathbb{N}, \operatorname{Msg}]=\operatorname{Map}[$ for $(n<-\mathbb{N}$ ) yield $(n, \emptyset)] / /$ buffered messages (asynchrony)

// Auxiliary Methods

def processReceive(message: Msg ) \{ // Receiving a message

if $(\forall \mathrm{m} \in$ mbox. $\mathrm{m}$. sender $\neq$ message. sender $)\{/ /$ check duplication (UDP or byzantine) mbox $=$ message $::$ mbox

round[currentRound]. receive(message.sender, message.payload) checkProgress()

\} \}

def checkProgress() \{ // Update timeout and strict according to Progress

if (Progress.isTimeout) $\{$ timeout $=$ Progress.timeout; strict $=$ ! Progress. catchUp $\}$

else if (Progress.isWaitMessage) $\{$ timeout $=\infty$; strict $=$ true $\}$

else if (Progress.isGoAhead) $\{$ strict $=$ false; nextRound $=\max ($ nextRound, currentRound +1$)$ \}

if (Progress. syncNb > 0 ) \{

if (maxRound.values.filter ( $>>=$ currentRound). size $>=$ Progress. syncNb $+F$ ) \{ strict $=$ false $\}$

else $\{$ timeout $=\infty$; strict $=$ true $\}$

\} \}

def $\operatorname{catchUpTo}()=$ maxRound values. $\operatorname{sorted}[N-F-1] / /$ highest round excluding byzantine processes

//Execute the program, each iteration of the loop corresponds to one round

while(true) \{

// SEND

for ( (dest, payload) <- round[currentRound].send())

Network.sendTo(dest, Msg(id, currentRound, payload, retransmit))

// RECEIVE INIT

strict $=$ false; $\operatorname{mbox}=[] / /$ clean

roundStart = currentTime ()$/ /$ set time

round[currentRound]. receiveInit()

checkProgress()

// RECEIVE

for ( message <- pendingMessages[currentRound] ) processReceive(message) // deliver buffered msg

while ( $($ nextRound $==$ currentRound $\vee$ strict $)$ ) $\{/ /$ not enough messages received

message $=$ Network. receiveWithTimeout $($ roundStart + timeout - currentTime ()$)$

if (message $==$ null) $\{/ /$ timeout

strict $=$ false

nextRound $=\max ($ nextRound, currentRound +1$)$

\} else \{

$\operatorname{maxRound}[$ message. sender $]=\max (\max$ Round [message. sender $]$, message. round $)$

if (message. round < currentRound) \{\}$/ /$ late message, ignore

else if (message. round $==$ currentRound) $\{$ processReceive(message) $\}$

else \{ pendingMessages[message. round]. add(message) // buffer and

nextRound $=\max ($ nextRound, catchUpTo ()$)\} / /$ check if catching-up is needed

\} $\}$

// FINISHROUND

round[currentRound]. finishRound(mbox)

currentRound $+=1$; $\operatorname{maxRound}[\mathrm{id}]=$ currentRound

nextRound $=\max ($ nextRound, currentRound $)$

\}

Proc. ACM Program. Lang., Vol. 4, No. OOPSLA, Article 213. Publication date: November 2020. 
of catch-up uses two variables nextRound, ranging over round numbers, and strict which is a boolean mirroring Progress. catchUp.

In the byzantine case, i.e., $F>0$, it is not sound for a process to catch-up upon a single message reception, because a Byzantine process may send arbitrary round numbers. Therefore, the runtime keeps not only the buffer of pending messages but also an array maxRound, that for each process stores the highest round value it sent in a message. Instead of jumping to the maximal round number, the runtime ignores the $F$ highest round values (line 30 ) in the array maxRound, and jumps to the $F+1$ st highest one (at least one correct process made progress until that round).

In case of a jump, the message accumulator of the current round stops, and finishRound is executed for the current and all the other rounds, up to nextRound.

Synchronization algorithm. Although all protocols may use Progress.sync(k), we mainly used this primitive in byzantine protocols, where $k$ equals $F+1$, for two phase commit (where $k=N$ ). It ensures that $k$ correct processes, i.e., non-byzantine and not-crashed, are synchronously switching at runtime the respective round.

The implementation of Progress. sync $(k)$ forces the runtime to wait for $F+k$ messages sent by different processes for the current round or any other higher round before terminating the current message accumulator, and do a round switch. This is done using the maxRound map which stores the highest round number received from each process. Over networks with reliable links, two timeouts ensure that between round switches the waiting time is long enough to let correct process exchange messages. However, over networks with lossy links timeouts are an underestimation. The transport layer is responsible for resending the messages of the round, and therefore, the synchronization algorithm an infinite timeout assuming the transport layer will eventually deliver enough messages. The implementation of this synchronization primitive is possible in the round-based model only under reliable networks, i.e., TCP. Resending the messages of a round breaks the round structure, which requires the number of sent messages to be bounded by the state of the process and the number of processes in the network. Therefore, it needs to be done either by the runtime or the transport layer (reprensented by the boolean flag retransmit). Also, over networks with lossy links it is necessary to consider messages from higher rounds. A message that is from a round $f r$ greater than the current round $c r$, witnesses that the process which sent it was at some point in $c r$, therefore it will be counted among the $2 F+1$ messages (from different processes) required to validate the synchronization barrier. This behavior it is not possible implementable directly in the round model.

The runtime uses an auxiliary method processReceive to check that only one message per process per round is delivered. This deals with malicious processes which may send multiple messages and link faults, e.g., UDP may duplicate packets.

\subsection{Runtime Correctness}

At runtime, the executions are asynchronous, i.e., processes may be in different rounds at the same time, and processes may receive messages coming from rounds that are different from their current one. We show that these runtime asynchronous executions are indistinguishable from the RESYNC lockstep semantics.

Definition 5.1 (Indistinguishability). Two executions $\pi$ and $\pi^{\prime}$ are indistinguishable w.r.t. a set of actions $A$, denoted $\pi \simeq_{A} \pi^{\prime}$, iff for every process $p$, the projection of both executions on $p$ and on the actions in $A$ agree up to finite stuttering.

Next, by send, receiveInit, receive, and finishRound, we refer to the execution, at runtime, of the wrappers of those respective methods in RESYNC program. 
Theorem 5.2 (Correctness). Given a RESYNC program $\mathcal{P}$, for every execution ae $\in\left[[(\mathcal{P})]_{\text {Run }}\right.$, there exists an indistinguishable execution se $\in[[\mathcal{P}]]$ with respect to the actions $C=\{$ init, send, receiveInit, receive, finishRound $\}$, that is, ae $\sim_{C}$ se.

Proof SKetch. Let us consider an asynchronous execution ae $\in\left[[(\mathcal{P})]_{\text {Run }}\right.$. The main ingredient is that the runtime only provides messages for the current round to the RESyNC program. Thus, reduction arguments [Chaouch-Saad et al. 2009; Damian et al. 2019; Elrad and Francez 1982] due to communication closure allow us to reduce to indistinguishable executions where globally actions are ordered with non-decreasing round numbers. Then, we use the fact that send and receive are left and right movers [Lipton 1975], resp. This generates indistinguishable executions that have all the init and send as well as the finishround for the same round appearing in a block, so that they can be reduced to synchronized actions as required for se $\in[[\mathcal{P}]$. Timeout actions to (and additional sends for byzantine rounds) in the asynchronous executions lead to stuttering invisible events, which we may drop and maintain indistinguishability.

Theorem 5.2 implies that the specifications closed under indistinguishability are preserved by the execution platform. These specifications (also called local properties in [Chaouch-Saad et al. 2009]) form an important class, which includes consensus, k-set agreement, and leader election.

For networks that are partially synchronous we prove a stronger result. Partially synchrony is defined over the physical model of the network, with immediate consequences over the runtime executions, but also over the high-level round-based executions. Let $T(m)$ be the time it takes between the moment a message $m$ is added to the send pool sms and the moment it either gets dropped by the network or it is delivered to its recipient. A (physical) network is partially synchronous if there exists a $\Delta$, and a global stabilization time GST, such that the transmission delays of all messages $m$ sent after GST from a correct processes to a correct process satisfy $T(m)<\Delta$. The original definition in [Dwork et al. 1988] assumes also that a bound on the relative speed of processes holds only after GST. However, on modern architectures this bound holds during the entire computation. Note that before GST arbitrarily many messages might be dropped.

In the round-based model, the existence of GST means that from some round on, called GSR, all messages sent by correct processes to correct processes are received. Prior to GSR arbitrarily many messages may be dropped. Given a program $\mathcal{P}$, the set of partially synchronous lockstep executions of $\mathcal{P}$ is a subset of $[[\mathcal{P}]]$, consisting only in those executions where there exists global synchronization round GSR.

Theorem 5.3 (PS Correctness - Closed). Given a RESYNC program $\mathcal{P}$ with closed rounds, for every execution ae $\in\left[[(\mathcal{P})]_{\text {Run }}\right.$ over a partially synchronous network, there exists an indistinguishable partially synchronous execution pse $\in[[\mathcal{P}]]$ w.r.t. the common actions $C=\{$ send, receiveInit, receive, finishRound $\}$, that is, ae $\sim_{C}$ pse.

The proof of Theorem 5.3 follows similar ideas as the proofs in [Dwork et al. 1988]. In the following, we emphasize a few crucial differences that make the execution platform of $\mathrm{RESYNC}$ practical. A distributed clock is constructed [Dwork et al. 1988], which ensures that at least $F+1$ correct processes have closely synchronized local estimates of the clock. The distributed clock has two purposes: (i) it ensures that after GST all correct processes will quickly resynchronize and (ii) a process uses the clock ticks to determine how long to wait for the messages of a round. In [Dwork et al. 1988], at every process, the steps of the distributed clock algorithm are interleaved with the steps of the consensus protocol that is executed. During a round of the consensus algorithm, the clock ticks multiple times, as the clock is used to burn time and slow down fast processes (to wait sufficiently long for messages). Each tick is implemented by an instance of a distributed clock synchronization scheme, which involves all-to-all communication. In practice this would flood the 
network with many messages and slow down the consensus computation (due to interleavings of the clock with the algorithm).

Instead of using a distributed clock to timeout messages, the runtime of RESYNC uses a hardware clock, and it implements a distributed clock only to synchronize the round start, that is, it uses only one clock value per round, contrary to many clock ticks in [Dwork et al. 1988]. By replacing many distributed clock ticks with a hardware clock RESYNC's runtime gives the programmer the possibility to use timeouts that 1 ) estimate the time it takes for the slowest correct process to resynchronize with the fastest correct process and 2) estimate the time to process and transmit a message.

Definition 5.4 (Observational refinement). Given two transition systems TS1 and TS2 and a set of actions of the two systems $O$, called observable actions, TS1 observationally refines TS2, denoted $T S 1 \triangleright_{O} T S 2$, iff all executions of TS1 projected on the observable actions $O$ are included in the executions of TS2 projected on the observable actions $O$.

The observable actions of a RESync program are calls to the interface methods. The runtime executions and the lockstep executions are w.r.t. the interface actions. In [Filipovic et al. 2009] sequentially consistency is proven equivalent with observational refinement for commutative clients, i.e., when the interface operations commute. This proof is adapted straightforward to indistinguishability.

COROLLARY 5.5. Given a program $\mathcal{P}$ the runtime semantics of $\mathcal{P}$ observationally refines the RESYNC semantics of $\mathcal{P}$ w.r.t. the actions $O$ in the interface of $\mathcal{P}$, if the interface actions commute for the client, that is, $[[(\mathcal{P}[\text { true }])]]_{\text {Run }} \triangleright_{O}\left[[\mathcal{P}[\right.$ true $]]$ and $[[(\mathcal{P}[P S])]]_{\text {Run }} \triangleright_{O}[[\mathcal{P}[P S]]]$.

\subsection{Runtime Implementation Details}

The runtime implements the algorithm in Listing 1 . We discuss in this section aspects related to the message structure and memory management, which are not explicitly visible in the algorithm in Section 5 as its presentation focuses on the computation.

Typed rounds and serialization. From the user's perspective, the communication is typed, i.e. the rounds specify the type of the message payload. All the serialization is encapsulated within the rounds. The runtime itself is agnostic to the message content, it just moves bytes.

Since we consider Byzantine processes, serialization is a weak point and can often be abused by malicious processes. While hardening against that type of attack is outside the scope, we take basic protection measure. We use $\mathrm{KRYO}^{2}$ for the serialization which requires explicit registration of the types which can be deserialized. While we provide safe serializers for simple types, e.g, collections of primitive types, any serializer for more complex objects needs to be provided by the user and properly hardened.

Self messages bypass. Sending a message to self would be wasteful. RESYNC detects these messages and handles them separately. In particular, these messages are kept within the round itself and directly forwarded to the receive method. This makes it possible to keep the program simple, i.e., treat the current processes just as another process, without incurring any extra cost.

Memory management aspects. One important aspect when implementing the runtime is keeping the maximal amount of used memory bounded. Bounding the memory is essential when considering malicious attackers. Since we store messages that are supposed to be delivered later, in the buffer pendingMessages, a malicious process could send many messages with very high round number

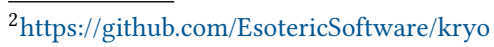


and thus make pendingMessages grow arbitrarily large. Therefore, we put a bound on the maximal number of incoming messages per process that are stored. When there are too many messages, we evict the ones with the smallest round number.

Reallocating all the data structures required by the runtime, e.g., maxRound, pendingMessages, with each new program execution, causes an important performance loss. Therefore, we pool and reuse the objects with a complex internal structure as well as the memory buffers for sending and receiving messages.

Round number and overflow. During an execution the round number can grow arbitrarily. While we could use arbitrary precision number to account for that grow, we decided to implement numbers with finite precision and take advantage of the wrap around semantics of integers on the JVM. This means a comparison $a \geq b$ becomes $a-b \geq 0$. The advantage of this solution is its simplicity and efficiency. Theoretically speaking, it is only correct as long as the distance between the fastest and slowest correct processes is smaller than half of the range of an int $\left(2^{31}-1\right)$.

\section{EXPERIMENTAL EVALUATION}

We have implemented RESYnC in ScAla on top of the PSync [Drăgoi et al. 2016] codebase and evaluated it experimentally. The goal of the experiments is to 1) compare the performance RESYNC with largely-used distributed systems 2) compare the expressiveness and performance of RESYNC with other domain specific languages, especially with PSYNC, and 3) compare different RESYNC implementations of the same theoretical algorithm.

We run our experiments on servers with 2 Intel Xeon X5650 ( 6 cores at $2.67 \mathrm{GHz}$ ), 48GB of RAM, and a gigabit network interface. The average ping between any two machines is around $0.17 \mathrm{~ms}$. The servers run Debian stretch with Linux kernel 4.14 and we use the OpenJDK 11. As the JVM has a slow startup and the runtime of RESYNC allocates most resources at the beginning, we report averages over runs of 5 minutes. The implementation is available at https://github.com/dzufferey/psync and a explanation on how we run the experiments is available at https://github.com/dzufferey/resync oopsla20_artifact. The RESynC model is agnostic to the transport layer. We support TCP and UDP in the benign case. Authentication for Byzantine protocols is implemented using TLS on top of TCP ${ }^{3}$. This also protects from a large class of Sybil and replay attacks. Being able to precisely identify the sender and only receive from expected senders is necessary for the integrity of the model.

\subsection{Throughput Evaluation}

We perform two experiments comparing the throughput of consensus algorithms in RESYNC against widely used systems solving consensus. The first experiment looks at the case of benign faults while the second one compares Byzantine fault-tolerant implementations.

Benign consensus. RESync is evaluated on an iterated version of Paxos (multi-Paxos), that uses an adaptation in RESYNC of round-based version of Paxos given by [Charron-Bost and Schiper 2009], called LastVoting. To this, we implemented a message accumulator where the leader progresses as soon as it receives a majority of votes and the acceptors progress after receiving a message from the leader. The algorithm is used in a key-value store. Each decision reached by an instance of the consensus algorithm (LastVoting) handles a batch of requests. The batch contains $32 \mathrm{~KB}$ of data. We measure the amount of data that the system can process per second. The results are shown in Fig. 12a. The throughput of the system is measured in MB per second. To test the scalability of the system, we run the test on 3 to 9 replicas.

\footnotetext{
${ }^{3}$ The user of RESynC still needs to provide the mechanism used to authenticate processes, e.g. check certificates.
} 


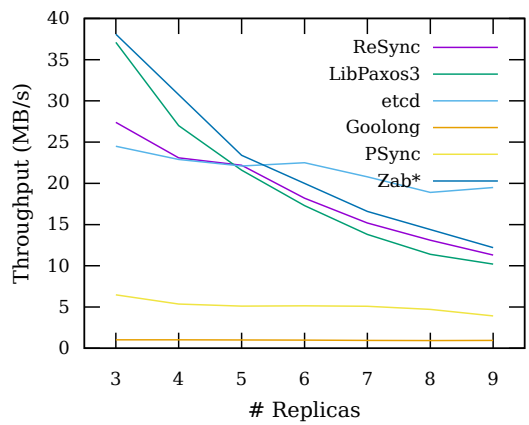

(a) Benign test: ReSYNC against LiBPAXos3, ETCD, Goolong and ZAB

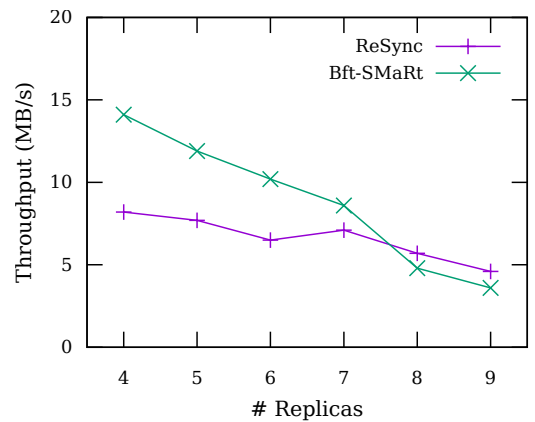

(b) Byzantine test: RESYNC against BFT-SMART

Fig. 12. Comparison for benign and Byzantine Consensus Algorithm

We compared the ReSync implementation of consensus with LiBPaxos3 [Sciascia 2016], ETCD [etc 2019], Goolong [von Gleissenthall et al. 2019], PSync [Drăgoi et al. 2016], and ZAB [Junqueira et al. 2011]. LiBPAxos3 is a $\mathrm{C}$ implementation of multi-Paxos. The system processes requests which are simple arrays of bytes. We use requests size of $32 \mathrm{~KB}$ to match the size of a batch in RESYNC. ETCD version 3.4.3, is a Go implementation of the Raft algorithm by [Ongaro and Ousterhout 2014] We use the provided benchmarking tool with 1000 clients making request of $32 \mathrm{~KB}$. With more clients the throughput stops increasing and the system becomes unstable. Goolong is a multi-Paxos written in Go. It is also used in a key-value store and processes requests in batches of $100 \mathrm{~KB}$ of data PSYNC also implements the Last Voting algorithm but instead of a message accumulator it waits until it reaches the timeout. We also include the performance numbers from Zab [Junqueira et al. 2011] taken from the paper as a baseline reference. ${ }^{4}$ Overall, RESync follows the behavior exhibited by LiBPAXOs3 and ZAB which are production systems used in industry. With a smaller number of replicas, we can see the cost of the round abstraction. With more replicas, this cost disappears as more messages help RESYNC keep a tighter synchronization between processes. ETCD and GoolONG behave differently. ETCD uses a compression layer which penalizes the system with few replicas. On the other hand, it is not strongly limited by the network. For 8 and 9 replicas it exceeds what the network bandwidth allows without compression. GoolONG is CPU bound independently of the number of replicas

Byzantine consensus. We have implemented in RESYnC the normal decision algorithm from PBFT [Castro and Liskov 2002]. The algorithm uses a leader which sends requests to all the replicas. Then all the replicas perform two rounds of all-to-all communication to establish and confirm a quorum larger than $2 n / 3$ around that request. During the all-to-all rounds, only digests instead of the full requests are forwarded. We use SHA-256 to compute the digest of the request. We compare our implementation against BFT-SMARt [Bessani et al. 2014]. BFT-SMART is a high-performance Byzantine fault-tolerant state machine replication library developed in Java. This library implements a protocol similar to PBFT together with complementary protocols to boost performance. The results are shown in Fig. 12b. Similar to the benign case, RESync has a higher overhead for small numbers of replicas. The overhead tappers off as the number of replicas increases. On the other

\footnotetext{
${ }^{4}$ Benchmarking distributed algorithms fairly is difficult. Each system has many parameters which can be tuned to achieve the best performances. Therefore, we want to include a baseline comparison with a system tuned by its authors. To make the comparison fair, we run the test on old machines that match the evaluation of Zab performed in 2011 [Junqueira et al 2011]. The system has very likely been evaluated on Intel Xeon X5630. The X5630 processors compared to the X5650 have 4 cores instead of 6 and run at $2.53 \mathrm{GHz}$ instead of $2.67 \mathrm{GHz}$.
} 
hand, RESync has a larger synchronization overhead due to the synchronization primitive that forces $F+1$ processes to stay synchronized, which is more visible with a small number of processes. An interesting point to observe is the transition between 6 and 7 replicas for RESYNC: 6 replicas tolerate 1 fault and 7 replicas tolerate 2 malicious processes. In both case, 5 messages are expected for a quorum. Therefore, if some replicas are slow, e.g., due to garbage collection, it has less impact as RESYNC waits for the first 5 messages.

\subsection{Comparison with PSYnc}

Table 2. Expressivity of RESYnC versus PSYNC

\begin{tabular}{|c|c|c|c|c|}
\hline Protocol & faults & PSYNC & RESynC & Message accumulator \\
\hline LastVoting & benign & $\checkmark$ & $\checkmark$ & Progress.timeout Progress.catchUp(true) \\
\hline One third rule & benign & $\checkmark$ & $\checkmark$ & Progress.timeout Progress.catchUp(true) \\
\hline Two-phase commit & benign & $x$ & $\checkmark$ & Progress.waitMessages Progress.catchUp(false) \\
\hline Failure detector & benign & $x$ & $\checkmark$ & Progress.timeout Progress.catchUp(false) \\
\hline$\Theta$-Model & benign & $x$ & $\checkmark$ & Progress.timeout Progress.catchUp(false) \\
\hline Game of life & benign & $x$ & $\checkmark$ & Progress.waitMessages Progress.catchUp(false) \\
\hline ViewChange & byzantine & $x$ & $\checkmark$ & Progress.sync $(F+1)$ Progress.catchUp(true) \\
\hline NormalOp & byzantine & $x$ & $\checkmark$ & Progress.sync $(F+1)$ Progress.catchUp(true) \\
\hline
\end{tabular}

We compared the expressiveness and performance of RESYNC with PSYNC whose runtime we build on top of. Table 2 shows protocols that are implementable in RESYNC and PSYNC. Last Voting by [Charron-Bost and Schiper 2009] (the round version of Paxos), one third rule by [Charron-Bost and Schiper 2009], and two-phase commit are consensus protocols. We implement the strong failure detector by [Chandra and Toueg 1996] and the perfect failure detector in the $\Theta$-Model by [Widder and Schmid 2009]. ViewChange and NormalOp are protocols from the round-based version of PBFT by [Milosevic et al. 2009]. We highlight for each protocol the constructs used to implement the message accumulator. In PSYNC the programmer can vary only the timeout, which restricts the set of protocols implementable in PSync.

Fig. 12a includes a performance comparison between the implementation of Paxos in RESYNC and the one in PSYNC. Although the core algorithm implemented in the two domain specific languages is the same-Last Voting [Charron-Bost and Schiper 2009]-the evaluation shows the advantage of using ReSync. Implementing a message accumulator gives an average speed up of $3.5 \times$. We see that the performance curve of PSYNC is much flatter and the progress towards agreeing on a decision value is limited as the rounds end with a timeout. To get the maximal performance, we find the minimal timeout value which allows the proposer to receive enough replies to form a quorum. With 3 replicas the timeout is $3 \mathrm{~ms}, 4-7$ replicas use $4 m s$, and $5 m s$ for 9 replicas. With a message accumulator, RESYNC does not need such tuning and can progress much faster.

There are no other domain specific languages that allow programming algorithms tolerant to both benign and byzantine faults. For the benign case we have experimented with DisTALGo [Liu et al. 2012]. However, their multi-Paxos implementation keeps the whole log of operations as a set in memory. The access gets slower as the algorithm progresses. Thus, we could not get performance measurement on longer runs as the algorithm stops making progress.

\subsection{Comparing the Effect of Deployment Conditions on Message Accumulators}

We performed more tests on the implementation of our runtime by evaluating the performance of benign Paxos and two-phase commit using different message accumulators. In these experiments, rather than maximizing the throughput using large requests which saturate the network, we try to generate many small packets which increase the load on the RESYNC runtime. We report the speed of the system by the number of requests where each request is processed independently. 


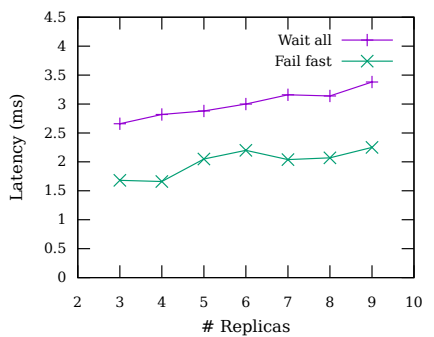

(a) Comparing progress conditions for the two-phase commit protocol with TCP and a $5 \mathrm{~ms}$ timeout

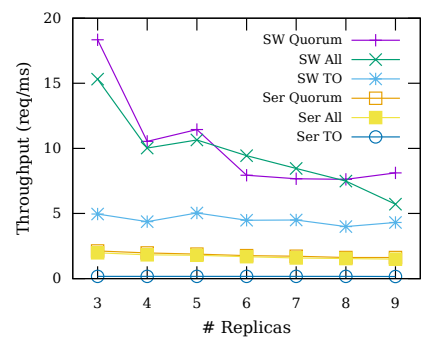

(b) Comparing progress conditions in Paxos with TCP transport and a 5 ms timeout

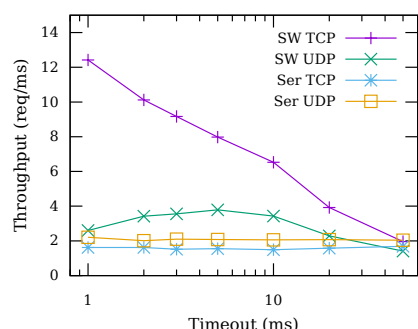

(c) Effect of timeout values and transport layer in Paxos with 9 replicas progressing on quorum

Fig. 13. Comparing progress conditions with the Paxos algorithm and the two-phase commit protocol

Two-phase commit. The two-phase commit protocol is interesting as the message accumulator inspects the received value. The protocol succeeds only if all the replica agrees. In Fig. 13a, we compare two message accumulator one which waits for all the replies before processing them in bulk and another one which terminates on the first negative reply. We can observe that the latency to process a request drops by one third when RESYNC inspects the message content.

Paxos. We use Paxos in two different scenarios of deployment.

Serializability (Ser): The system calling our consensus implementation, ensures the serializability of decisions reached by different consensus instances. Only when one consensus instance has terminated the next consensus instance starts.

Sliding Window (SW): We use a sliding window to increase the system load. While a decision is still pending in a consensus instance, the system already starts the next consensus instance. The size of the sliding window is the limit on how many decision can be performed in parallel. Decisions can happen out-of-order but they are reordered before being applied to the system. We use a sliding window of size 20 .

Waiting on some messages, all messages, or the timeout. We modified our Paxos implementation to test how different waiting conditions affect the algorithm. The replicas progress as soon as they receive message from the leader. On the other hand, the leader progresses (switches rounds) either when it received messages from a quorum or timeout (Quorum), or when it received messages from all the replicas or timeout (All), or on timeout (TO). The results are shown in Fig. 13b.

We see that the (TO) approach, which coincides with the one used in PSYNC, is much slower than the rest. For (Quorum) and (All), we can observe some interesting behaviors. The throughput is not monotonically decreasing with the number of replicas. These behaviors can be explained by the waiting condition of the message accumulator: To switch rounds and make progress towards the agreement, the leader needs to receive messages from a quorum of processes which includes the leader itself. So for 3 replicas, the 1st out of 2 messages leads to progress. For 4 replicas, the leader needs to receive 2 out of 3 messages and, for 5 replicas, 2 out of 4 messages. So each time we transition from an odd to even number of replicas the leader waits for one more message. When going from an even to odd number of replicas, the leader waits for the same number of messages but there is one more process sending. This effect is particularly pronounced when looking at the (Quorum) tests and, in a lesser extend, is also visible in the (All) tests.

Varying the timeout. One critical parameter is finding an appropriate timeout value for the duration of a message accumulator and implicitly for the duration of a round, which influences the synchronization of rounds across processes. Compared to other approaches where a timeout is 
used only to detect crashes, it may be better to not be conservative when picking the timeout in ReSync. ReSync's round synchronization primitives, catchUp and sync, are determined by the received messages, and timing out early results in several retries hence in sending more messages to synchronize the system.

Fig. 13c shows what happens when running Paxos on 9 replicas for different timeout values over either TCP or UDP. For this test, we use timeout values of $1,2,3,5,10,20$, and $50 \mathrm{~ms}$. Recall that the network latency is $0.17 \mathrm{~ms}$ and RESYNC timeout granularity is $1 \mathrm{~ms}$. In the Ser conditions, the system is sightly faster over UDP than TCP. On the other hand, with a higher load TCP has a clear advantage. With higher load, we can observe that the performance starts degrading when the timeout gets below $5 \mathrm{~ms}$ with UDP, while TCP makes a much better job at keeping the system synchronized with a small timeout. The TCP layer is highly-optimized, includes congestion control, and message retransmission. As TCP is lower down the networking stack, it can retransmit message more efficiently. At the application layer, the retransmission is more costly.

\section{RELATED WORK AND CONCLUSION}

Asynchronous programing languages. Programming languages for distributed systems have been developed recently with the goal of formal guarantees: the language P [Desai et al. 2013] for asynchronous event-driven applications has a dedicated specification language and a testing tool for concurrency bugs. The language $\mathrm{P}$ simplifies the asynchronous control structure. It would be interesting in the future to combine the two approaches (RESYNC and P) using for example P like structures to program the message accumulators.

Ivy [Padon et al. 2016] is a framework that uses a high-level language to specify and implement systems, and do verification. Protocols in Ivy are written in a general protocol language, using an event-based asynchronous semantics, out of which an EPR specification of the systems is extracted and executed under certain conditions. RESYNC takes a different approach: it identifies synchronization primitives inherent to fault-tolerance. These programing concepts can be used by engineers who want to fine-tune their implementations or verification tools alike.

DistAlgo [Liu et al. 2012] is a domain specific language that focuses on synchronization primitives (and not on verification). It uses quantified queries to express synchronization conditions. DistAlgo mixes asynchronous programming and formal specifications as it uses quantified queries to express synchronization conditions. In contrast, RESYNC proposes new synchronization primitives that alleviate the programmer from implementing systems using quantifiers.

There are also languages like Go and Erlang which are designed for programming in the distributed setting, or Rust which focusses on memory safety.

However, all these languages are either rooted in general programming languages or too focused on verification, and lack synchronization primitives that are inherent to fault-tolerance, such as an abstract notion of logical time [Fidge 1991].

Synchronous programming abstractions have been developed for simplifying programming distributed, concurrent and parallel systems. The most prominent are virtual synchrony [Birman and Joseph 1987], bulk synchronous parallelism [Valiant 1990] or phasers [Shirako et al. 2008]. Virtual synchrony [Birman and Joseph 1987] gives the illusion of synchrony using group communications [van Renesse et al. 1996]. Virtual synchrony does not allow custom optimization algorithms, and does not have synchronization primitives for byzantine protocols. Similarly, bulk synchronization uses stronger synchronization primitives and applies to parallel programs.

Round-based programming languages. Synchronous round-based models [Lynch 1996], with their inherent logical time provided by the round number, relieve the programmer from implementing any notion of logical time. Partially synchronous round-based models [Charron-Bost and Schiper 
2009; Dwork et al. 1988; Gafni 1998] were the inspiration and the main motivation of this work. Our programming abstraction extends round-based models by allowing the programmer to implement custom optimizations for the round-switch, addressing one of the main concerns raised w.r.t. the performance of round structures.

The closest related work is PSync [Drăgoi et al. 2016] that we build upon. Psync is a programming language based on a classic round based model, called Heard-Of [Charron-Bost and Schiper 2009]. Psync focuses on formal verification and execution of a strict subset of the partially-synchronous systems captured by the Heard-Of model. For example, two-phase commit is not implementable in PSYNC, although other consensus protocols are. Two-phase commit is designed for synchronous systems which requires that all messages sent in a round should be delivered in the round, which is not guaranteed by PSYNC. PSYNC guarantees this only eventually, that is, from some round on. The runtime of PSYNC is rigid and cannot be customized to accommodate protocol-specific requirements regarding the received messages. Contrary to PSYNC, or any other round model, RESYNC proposes a new programming abstractions where round boundaries are under the control of the programer instead of a restricted runtime. RESYNC can handle byzantine faults, which is not the case of PSYNC, and the new synchronization primitives are essential to achieve this. In [Bravo et al. 2020] the authors introduce a synchronizer that is also motivated by the classic partially synchronous roundbased model [Dwork et al. 1988]. This synchronizer offers an alternative to the runtime algorithm for closed rounds as it allows a more coarse grain protocol specific synchronization.

Verification perspective. Although RESync has a round structure, which is known for simplifying the verification task, the focus of this paper is on overcoming the limitations of round-structures and the performance pitfalls which arise when verification is the primary goal of a domain specific language. Formal verification of RESYNC programs is orthogonal and goes beyond the ambitions of this paper. However, the improvement of performance does not make the verification task more difficult. Rather, it allows a more structured two-step verification approach: (i) verify a roundbased algorithm and (ii) verify the implementation of the round boundaries. Regarding (i), RESYNC preserves the benefits of PSYNC, and more general of classic round structures, in proving safety properties for systems where network assumptions are required only to ensure liveness (like Paxos). This allows simpler proofs, i.e., invariants, due to a reduced number of interleavings compared with asynchronous computational models. Moreover, for a fragment of RESYNC (closed rounds) existing verification techniques [Aminof et al. 2018; Charron-Bost and Merz 2009; Debrat and Merz 2012; Dragoi et al. 2014; Marić et al. 2017; Stoilkovska et al. 2019] developed for round-based systems apply, and some of them also cover some byzantine protocols. However, we leave a verification engine for RESYNC as future work, as it requires not only integrating known results and addressing (ii) Specifying these combinations can be done by adapting the so called communication predicates in the Heard-Of model but the proof rules are future work.

Conclusion. RESYNC is a new programming abstraction for fault-tolerant distributed protocols. It proposes the first round structure with parametrized round switching policies, making the performance of the round-based code adaptable to the networks the programs are written for.

\section{ACKNOWLEDGMENTS}

We thank the OOPSLA reviewers and Alexey Gotsman for useful comments and suggestions. Damien Zufferey is supported in part by the Deutsche Forschungsgemeinschaft project 389792660-TRR 248 and by the European Research Council under the Grant Agreement 610150 (http://www.impacterc.eu/) (ERC Synergy Grant ImPACT). Most of the work was done while Cezara Drăgoi was at INRIA, France, supported by the French National Research Agency ANR project SAFTA (12744ANR-17-CE25-0008-01). Cezara Drăgoi and Josef Widder are supported by Interchain Foundation. 


\section{REFERENCES}

2019. etcd. Retrieved Nov 21, 2019 from https://etcd.io/

Benjamin Aminof, Sasha Rubin, Ilina Stoilkovska, Josef Widder, and Florian Zuleger. 2018. Parameterized Model Checking of Synchronous Distributed Algorithms by Abstraction. In VMCAI. 1-24.

Mathieu Baudet, Avery Ching, Andrey Chursin, George Danezis, François Garillot, Dahlia Malkhi Zekun Li, Oded Naor, Dmitri Perelman, and Alberto Sonnino. 2019. State Machine Replication in the Libra Blockchain. https://developers. libra.org/docs/assets/papers/libra-consensus-state-machine-replication-in-the-libra-blockchain.pdf.

Alysson Neves Bessani, João Sousa, and Eduardo Adílio Pelinson Alchieri. 2014. State Machine Replication for the Masses with BFT-SMART. In 44th Annual IEEE/IFIP International Conference on Dependable Systems and Networks, DSN 2014, Atlanta, GA, USA, June 23-26, 2014. IEEE Computer Society, 355-362. https://doi.org/10.1109/DSN.2014.43

Martin Biely, Bernadette Charron-Bost, Antoine Gaillard, Martin Hutle, André Schiper, and Josef Widder. 2007. Tolerating corrupted communication. In PODC. 244-253.

K. Birman and T. Joseph. 1987. Exploiting Virtual Synchrony in Distributed Systems. SIGOPS Oper. Syst. Rev. 21, 5 (Nov. 1987), 123-138.

Manuel Bravo, Gregory Chockler, and Alexey Gotsman. 2020. Making Byzantine consensus live. International Symposium on Distributed Computing (2020), to appear.

Ethan Buchman. 2016. Tendermint: Byzantine Fault Tolerance in the Age of Blockchains. Master's thesis. University of Guelph. http://hdl.handle.net/10214/9769.

Ethan Buchman, Jae Kwon, and Zarko Milosevic. 2018. The latest gossip on BFT consensus. CoRR abs/1807.04938 (2018). http://arxiv.org/abs/1807.04938

CASSANDRA 2013. Bug report. https://issues.apache.org/jira/browse/CASSANDRA-6023. accessed May 2020.

Miguel Castro and Barbara Liskov. 2002. Practical byzantine fault tolerance and proactive recovery. ACM Trans. Comput. Syst. 20, 4 (2002), 398-461. https://doi.org/10.1145/571637.571640

Tushar Deepak Chandra and Sam Toueg. 1996. Unreliable Failure Detectors for Reliable Distributed Systems. F. ACM 43, 2 (1996), 225-267.

Mouna Chaouch-Saad, Bernadette Charron-Bost, and Stephan Merz. 2009. A Reduction Theorem for the Verification of Round-Based Distributed Algorithms. In RP (LNCS), Vol. 5797. 93-106.

Bernadette Charron-Bost and Stephan Merz. 2009. Formal Verification of a Consensus Algorithm in the Heard-Of Model. Int. F. Software and Informatics 3, 2-3 (2009), 273-303.

Bernadette Charron-Bost and André Schiper. 2009. The Heard-Of model: computing in distributed systems with benign faults. Distributed Computing 22, 1 (2009), 49-71.

Tyler Crain, Vincent Gramoli, Mikel Larrea, and Michel Raynal. 2018. DBFT: Efficient Leaderless Byzantine Consensus and its Application to Blockchains. In 17th IEEE International Symposium on Network Computing and Applications, NCA 2018, Cambridge, MA, USA, November 1-3, 2018. 1-8.

Andrei Damian, Cezara Drăgoi, Alexandru Militaru, and Josef Widder. 2019. Communication-closed asynchronous protocols. In $C A V$. (to appear).

Henri Debrat and Stephan Merz. 2012. Verifying Fault-Tolerant Distributed Algorithms in the Heard-Of Model. Archive of Formal Proofs 2012 (2012).

Ankush Desai, Vivek Gupta, Ethan K. Jackson, Shaz Qadeer, Sriram K. Rajamani, and Damien Zufferey. 2013. P: safe asynchronous event-driven programming. In ACM SIGPLAN Conference on Programming Language Design and Implementation, PLDI '13, Seattle, WA, USA, June 16-19, 2013. 321-332.

Cezara Dragoi, Thomas A. Henzinger, Helmut Veith, Josef Widder, and Damien Zufferey. 2014. A Logic-Based Framework for Verifying Consensus Algorithms. In VMCAI, Kenneth L. McMillan and Xavier Rival (Eds.). Springer, 161-181.

Cezara Drăgoi, Thomas A. Henzinger, and Damien Zufferey. 2016. PSync: a partially synchronous language for fault-tolerant distributed algorithms. In POPL. 400-415.

Partha Dutta, Rachid Guerraoui, and Leslie Lamport. 2005. How Fast Can Eventual Synchrony Lead to Consensus?. In 2005 International Conference on Dependable Systems and Networks (DSN 2005), 28 fune - 1 July 2005, Yokohama, Japan, Proceedings. 22-27.

Cynthia Dwork, Nancy Lynch, and Larry Stockmeyer. 1988. Consensus in the Presence of Partial Synchrony. FACM 35, 2 (April 1988), 288-323.

Tzilla Elrad and Nissim Francez. 1982. Decomposition of Distributed Programs into Communication-Closed Layers. Sci. Comput. Program. 2, 3 (1982), 155-173.

Colin Fidge. 1991. Logical Time in Distributed Computing Systems. Computer 24, 8 (Aug. 1991), 28-33.

Ivana Filipovic, Peter W. O’Hearn, Noam Rinetzky, and Hongseok Yang. 2009. Abstraction for Concurrent Objects. In Programming Languages and Systems, 18th European Symposium on Programming, ESOP 2009, Held as Part of the foint European Conferences on Theory and Practice of Software, ETAPS 2009, York, UK, March 22-29, 2009. Proceedings (Lecture Notes in Computer Science), Giuseppe Castagna (Ed.), Vol. 5502. Springer, 252-266. https://doi.org/10.1007/978-3-642-

Proc. ACM Program. Lang., Vol. 4, No. OOPSLA, Article 213. Publication date: November 2020. 
00590-9_19

Michael J. Fischer, Nancy A. Lynch, and M. S. Paterson. 1985. Impossibility of Distributed Consensus with one Faulty Process. F. ACM 32, 2 (April 1985), 374-382.

Eli Gafni. 1998. Round-by-Round Fault Detectors: Unifying Synchrony and Asynchrony (Extended Abstract). In Proceedings of the Seventeenth Annual ACM Symposium on Principles of Distributed Computing, PODC '98, Puerto Vallarta, Mexico, fune 28 - fuly 2, 1998, Brian A. Coan and Yehuda Afek (Eds.). 143-152.

Flavio Paiva Junqueira, Benjamin C. Reed, and Marco Serafini. 2011. Zab: High-performance broadcast for primary-backup systems. In DSN. 245-256.

Ramakrishna Kotla, Lorenzo Alvisi, Michael Dahlin, Allen Clement, and Edmund L. Wong. 2009. Zyzzyva: Speculative Byzantine Fault Tolerance. ACM Trans. Comput. Syst. 27, 4 (2009), 7:1-7:39.

Avinash Lakshman and Prashant Malik. 2010. Cassandra: a decentralized structured storage system. Operating Systems Review 44, 2 (2010), 35-40.

Leslie Lamport. 2005. Generalized Consensus and Paxos. Technical Report. 60 pages. https://www.microsoft.com/enus/research/publication/generalized-consensus-and-paxos/

Richard J. Lipton. 1975. Reduction: A Method of Proving Properties of Parallel Programs. Commun. ACM 18, 12 (1975), 717-721.

Yanhong A. Liu, Scott D. Stoller, Bo Lin, and Michael Gorbovitski. 2012. From clarity to efficiency for distributed algorithms. In Proceedings of the 27th Annual ACM SIGPLAN Conference on Object-Oriented Programming, Systems, Languages, and Applications, OOPSLA 2012, part of SPLASH 2012, Tucson, AZ, USA, October 21-25, 2012, Gary T. Leavens and Matthew B. Dwyer (Eds.). ACM, 395-410. https://doi.org/10.1145/2384616.2384645

Nancy Lynch. 1996. Distributed Algorithms. Morgan Kaufman.

Ognjen Marić, Christoph Sprenger, and David A. Basin. 2017. Cutoff Bounds for Consensus Algorithms. In CAV. $217-237$.

Zarko Milosevic, Martin Hutle, and André Schiper. 2009. Unifying Byzantine Consensus Algorithms with Weak Interactive Consistency. In Principles of Distributed Systems, 13th International Conference, OPODIS 2009, Nîmes, France, December 15-18, 2009. Proceedings. 300-314.

Oded Naor, Mathieu Baudet, Dahlia Malkhi, and Alexander Spiegelman. 2019. Lumière: Byzantine View Synchronization. CoRR abs/1909.05204 (2019). arXiv:1909.05204 http://arxiv.org/abs/1909.05204

Brian M. Oki and Barbara Liskov. 1988. Viewstamped Replication: A General Primary Copy. In PODC. 8-17.

Diego Ongaro and John K. Ousterhout. 2014. In Search of an Understandable Consensus Algorithm. In 2014 USENIX Annual Technical Conference, USENIX ATC '14, Philadelphia, PA, USA, June 19-20, 2014., Garth Gibson and Nickolai Zeldovich (Eds.). 305-319. https:/www.usenix.org/conference/atc14/technical-sessions/presentation/ongaro

Oded Padon, Kenneth L. McMillan, Aurojit Panda, Mooly Sagiv, and Sharon Shoham. 2016. Ivy: safety verification by interactive generalization. In PLDI. 614-630.

Nicola Santoro and Peter Widmayer. 1989. Time is not a healer. In STACS (LNCS), Vol. 349. 304-313.

Nicola Santoro and Peter Widmayer. 2007. Agreement in synchronous networks with ubiquitous faults. Theor. Comput. Sci. 384, 2-3 (2007), 232-249.

Daniele Sciascia. 2016. LibPaxos3. Retrieved July 9, 2019 from https://bitbucket.org/sciascid/libpaxos/

Jun Shirako, David M. Peixotto, Vivek Sarkar, and William N. III Scherer. 2008. Phasers: a unified deadlock-free construct for collective and point-to-point synchronization. In Proceedings of the 22nd Annual International Conference on Supercomputing, ICS 2008, Island of Kos, Greece, June 7-12, 2008. 277-288.

T. K. Srikanth and Sam Toueg. 1987. Simulating Authenticated Broadcasts to Derive Simple Fault-Tolerant Algorithms. Distributed Computing 2, 2 (1987), 80-94. https://doi.org/10.1007/BF01667080

Ilina Stoilkovska, Igor Konnov, Josef Widder, and Florian Zuleger. 2019. Verifying Safety of Synchronous Fault-Tolerant Algorithms Bounded Model Checking. In TACAS, Part II (LNCS), Vol. 11428. 357-374

Pierre Sutra. 2019. On the correctness of Egalitarian Paxos. CoRR abs/1906.10917 (2019). http://arxiv.org/abs/1906.10917

Leslie G. Valiant. 1990. A Bridging Model for Parallel Computation. Commun. ACM 33, 8 (1990), 103-111. https: //doi.org/10.1145/79173.79181

Robbert van Renesse, Kenneth P. Birman, and Silvano Maffeis. 1996. Horus: A Flexible Group Communication System. Commun. ACM 39, 4 (1996), 76-83. https://doi.org/10.1145/227210.227229

Klaus von Gleissenthall, Rami Gökhan Kici, Alexander Bakst, Deian Stefan, and Ranjit Jhala. 2019. Pretend synchrony: synchronous verification of asynchronous distributed programs. PACMPL 3, POPL (2019), 59:1-59:30. https://doi.org/10. $1145 / 3290372$

Josef Widder and Ulrich Schmid. 2009. The Theta-Model: achieving synchrony without clocks. Distributed Comput. 22, 1 (2009), 29-47. https://doi.org/10.1007/s00446-009-0080-x

Maofan Yin, Dahlia Malkhi, Michael K. Reiter, Guy Golan-Gueta, and Ittai Abraham. 2019. HotStuff: BFT Consensus with Linearity and Responsiveness. In Proceedings of the 2019 ACM Symposium on Principles of Distributed Computing, PODC 2019, Toronto, ON, Canada, fuly 29 - August 2, 2019. 347-356. 
ZOOKEEPER 2017. Data Inconsistency bug report ZOOKEEPER-2832. https://issues.apache.org/jira/browse/ZOOKEEPER2832. accessed May 2020. 